\title{
Does Acute Caffeine Supplementation Improve Physical Performance in Female Team-Sport Athletes? Evidence from a Systematic Review and Meta-Analysis
}

\author{
Alejandro Gomez-Bruton 1,2,3,*iD, Jorge Marin-Puyalto 1,4 , Borja Muñiz-Pardos 1,2 , Angel Matute-Llorente 1,2,3 $\mathbb{D}^{1}$,

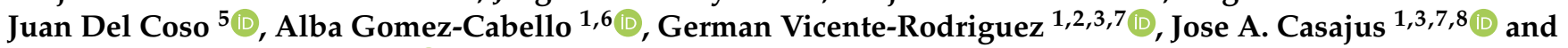 \\ Gabriel Lozano-Berges 1,2,3,7 (D)
}

1 GENUD (Growth, Exercise, Nutrition and Development) Research Group, FIMS Collaborating Center of Sports Medicine, 50012 Zaragoza, Spain; jmarinp@unizar.es (J.M.-P.); bmuniz@unizar.es (B.M.-P.); amatute@unizar.es (A.M.-L.); agomez@unizar.es (A.G.-C.); gervicen@unizar.es (G.V.-R.); joseant@unizar.es (J.A.C.); glozano@unizar.es (G.L.-B.)

2 Department of Physiatry and Nursing, Faculty of Health and Sport Sciences (FCSD), University of Zaragoza, 22001 Huesca, Spain

3 Physiopathology of Obesity and Nutrition Networking Biomedical Research Center (CIBERObn), 28029 Madrid, Spain

check for updates

Citation: Gomez-Bruton, A.; Marin-Puyalto, J.; Muñiz-Pardos, B.; Matute-Llorente, A.; Del Coso, J.; Gomez-Cabello, A.;

Vicente-Rodriguez, G.; Casajus, J.A.; Lozano-Berges, G. Does Acute Caffeine Supplementation Improve Physical Performance in Female Team-Sport Athletes? Evidence from a Systematic Review and Meta-Analysis. Nutrients 2021, 13, 3663. https://doi.org/10.3390/ nu13103663

Academic Editor: Ajmol Ali

Received: 7 September 2021 Accepted: 18 October 2021 Published: 19 October 2021

Publisher's Note: MDPI stays neutral with regard to jurisdictional claims in published maps and institutional affiliations.

Copyright: (c) 2021 by the authors. Licensee MDPI, Basel, Switzerland. This article is an open access article distributed under the terms and conditions of the Creative Commons Attribution (CC BY) license (https:// creativecommons.org/licenses/by/ $4.0 /)$.
4 Department of Physiatry and Nursing, Faculty of Health Sciences, University of Zaragoza, 50012 Zaragoza, Spain

5 Centre for Sports Studies, Rey Juan Carlos University, 28943 Fuenlabrada, Spain; juan.delcoso@urjc.es

6 Centro Universitario de la Defensa, University of Zaragoza, 50090 Zaragoza, Spain

7 Instituto Agroalimentario de Aragon IA2 (CITA-Universidad de Zaragoza), 50009 Zaragoza, Spain

8 Department of Physiatry and Nursing, Faculty of Medicine, University of Zaragoza, 50012 Zaragoza, Spain

* Correspondence: bruton@unizar.es; Tel.: +34-876553755

\begin{abstract}
Introduction: Recent original research and meta-analyses suggest that acute caffeine supplementation improves exercise performance in team-sport athletes (TSA). Nonetheless, most of the studies testing the effects of caffeine on TSA included samples of male athletes, and there is no meta-analysis of the performance-enhancing effects of caffeine on female TSA. The aim of the present study was to synthesize the existing literature regarding the effect of caffeine supplementation on physical performance in adult female TSA. Methods: A search was performed in Pubmed/Medline, SPORTDiscus and Scopus. The search was performed from the inception of indexing until 1 September 2021. Crossover randomized controlled trials (RCT) assessing the effects of oral caffeine intake on several aspects of performance in female TSA were selected. The methodological quality and risk of bias were assessed for individual studies using the Physiotherapy Evidence Database scale (PEDro) and the RoB 2 tool. A random-effects meta-analysis of standardized mean differences (SMD) was performed for several performance variables. Results: The search retrieved 18 articles that fulfilled the inclusion/exclusion criteria. Overall, most of the studies were of excellent quality with a low risk of bias. The meta-analysis results showed that caffeine increased performance in specific team-sport skills (SMD: 0.384, 95\% confidence interval (CI): 0.077-0.691), countermovement jump (SMD: 0.208, CI: 0.079-0.337), total body impacts (SMD: 0.488; 95\% CI: 0.050, 0.927) and handgrip strength (SMD: 0.395, CI: 0.126-0.665). No effects were found on the ratings of perceived exertion, squat jumps, agility, repeated sprint ability or agility tests performed after fatigue. Conclusions: The results of the meta-analysis revealed that acute caffeine intake was effective in increasing some aspects of team-sports performance in women athletes. Hence, caffeine could be considered as a supplementation strategy for female athletes competing in team sports.
\end{abstract}

Keywords: soccer; volleyball; basketball; ergogenic aid; elite athletes; sports performance 


\section{Introduction}

The use of caffeine in sporting events was controlled until 1 January 2004, since a post-competition urinary concentration above 12 micrograms per milliliter was considered an adverse analytical finding by the World Anti-Doping Agency [1]. However, at that date, caffeine was removed from the list of prohibited substances in the monitoring program of the World Anti-Doping Agency [2]. The removal of caffeine from the list of prohibited substances, in addition to increasing scientific knowledge about the potential ergogenic effects of caffeine, has caused an increase in caffeine intake in both men and women athletes over recent years [3].

The widespread use of this supplement in sport is based on scientific evidence, as it has been classified by the International Society of Sports Nutrition (ISSN) as a "Strong evidence to support efficacy and apparently safe" supplement [4], with recommended doses ranging from 3 to $6 \mathrm{mg}$ per $\mathrm{kg}$ of body mass with a timing of ingestion of $1 \mathrm{~h}$ before exercise. A vast amount of research indicates that caffeine intake can have a positive effect on several forms of athletic performance [5,6]. Grgic and colleagues performed an umbrella review in 2019 including 21 published meta-analyses, revealing that caffeine supplementation elicited an ergogenic effect on muscle endurance and strength, anaerobic power and aerobic endurance, which are critical variables for team-sports performance [5].

In team-sport athletes (TSA), the efficacy of caffeine supplementation in enhancing performance is less clear than in other sport disciplines, because success is explained by a combination of physical, technical and tactical skills. Brown et al. suggested via a meta-analysis that caffeine had no effect on repeated sprint ability (RSA) in TSA [7]. These results were contradicted by a review performed by Chia et al. [8], who found improvements in sprint performance (in 8 out of 10 studies) and vertical jump (in 7 out of 8 studies) in ball game athletes. These findings were reaffirmed by a later meta-analysis developed by Salinero et al. [9] evaluating TSA and also by a systematic review developed by Mielgo-Ayuso et al. [10] focusing on soccer players. Both studies concluded that acute caffeine ingestion improved jump height and RSA $[9,10]$ in addition to agility performance, total running distance and number of performed sprints during a match [9]. Nonetheless, Ferreira and colleagues [11] recently performed a meta-analysis focusing on the effects of caffeine on soccer, finding no significant improvements in soccer-related performance following caffeine supplementation. Therefore, although the positive effects that caffeine supplementation may have on athletic performance in certain individual sports (e.g., running, cycling, etc.) are evident, it seems that more research is needed to determine the ergogenic effect of acute caffeine intake in team sports.

Moreover, most of the studies included in the above-mentioned systematic reviews and meta-analyses only included male athletes, as stated in a recent letter to the editor by Salinero et al. entitled "More research is necessary to establish the ergogenic effect of caffeine in female athletes" [12]. In this letter, the authors analyzed the percentage of females in studies evaluating the ergogenic effects of caffeine, reporting that only $13 \%$ of the participants were female. Moreover, although some studies included both male and female participants (contributing to the aforementioned 13\%), most of them drew conclusions for the whole sample, irrespective of potential sex differences [13,14].

Despite the lack of research specifically analyzing female athletes, current guidelines for caffeine supplementation are identically applied for both males and females [15]. However, these guidelines were established primarily from studies developed in males, which is a clear limitation and raises concerns about their practicality. Although recent evidence suggests that the pharmacokinetics of acute caffeine intake seems to be similar in all phases of the menstrual cycle and that women athletes benefit from caffeine intake across all phases of the menstrual cycle [16], it is still possible that women obtain lower ergogenic effects of oral caffeine intake due to the interaction of caffeine and female sex hormones [17]. Along these lines, Temple and Ziegler [18] found sex differences in subjective and physiological responses to caffeine that were mediated by changes in circulating steroid hormones. In fact, inconsistent results have been found when comparing the ergogenic effects of 
caffeine in both sexes, with some studies finding some differences [19] while others found none $[20,21]$. Moreover, some researchers have concluded that the ergogenic effect of oral caffeine intake is present in both sexes but differs in its magnitude [22]. Along these lines, Mielgo-Ayuso et al. [10] recently developed a systematic review including 10 studies that evaluated the ergogenic effect of caffeine on both males and females. These authors concluded that caffeine supplementation produced a similar ergogenic benefit regarding aerobic performance and fatigue index in men and women, finding larger effects of caffeine intake in men when anaerobic performance was evaluated, which could be critical for team sports. However, the above-mentioned review only focused on the sex comparison; consequently, many studies that only recruited females were excluded, and due to the low number of included studies the authors chose not to perform a meta-analysis.

Thus, the aim of the present study is to perform a qualitative and quantitative analysis of the existing literature regarding the effect of caffeine supplementation on physical performance in adult female TSA.

\section{Methods}

\subsection{Search Strategy}

This systematic review and meta-analysis was carried out following the Preferred Reporting Items for Systematic reviews and Meta-Analyses (PRISMA) 2020 guidelines [23] and was pre-registered in PROSPERO (CRD42021223046). A systematic search was performed in the Pubmed/Medline, SPORTDiscus and Scopus databases. The search was performed from the inception of indexing until 1 September 2021, using the same search syntax as Salinero et al. [9] for Pubmed. An analogous search was performed for SPORTDiscus and Scopus (Supplementary Material: Table S1). All articles were downloaded to a CSV document to identify duplicates, and the whole process (i.e., identification, screening and selection of studies) was independently performed by two authors, with any disagreements resolved through discussion.

\subsection{Inclusion and Exclusion Criteria}

The following inclusion criteria were applied to selected studies: (1) studies evaluating the effect of an acute dose of isolated caffeine (e.g., not mixed with other supplements) on physical performance in female TSA (if studies included both sexes we only selected data for females, and if these data were not available we contacted the corresponding author and requested them); (2) studies including adults (18 years of age or over); (3) crossover studies that compared the intake of caffeine and a placebo; (4) studies using a blinded and randomized design; (5) studies in English or Spanish. Studies that supplied doses below $1 \mathrm{mg} / \mathrm{kg}$ or above $9 \mathrm{mg} / \mathrm{kg}$, that did not present a true placebo condition (thus not allowing for blinding) or that did not evaluate performance-related variables (e.g., only evaluated oxidative stress markers) were excluded. Note that performance-related variables are explained in detail in the "Data Extraction" section.

\subsection{Quality Assessment and Risk of Bias}

The Physiotherapy Evidence Database scale (PEDro) was used to evaluate the individual quality of each study, with studies being classified as excellent (score 9-10), good (score 6-8), fair (score 4-5) or poor (score $<4$ ). The PEDro scale has been shown to be valid and reliable for assessing the internal validity of randomized controlled trials [24].

Following the Cochrane Collaboration guidelines, the RoB 2 tool for randomized crossover designs was applied to assess the risk of bias of each study included [25]. RoB 2 includes the following domains for crossover trials: (1) bias arising from the randomization process; (2) bias due to deviations from the intended intervention; (3) bias due to missing outcome data; (4) bias in the measurement of the outcome; (5) bias in the selection of the reported results. Due to the characteristics of the crossover design, another domain related to bias arising from the period effect and the carryover effect should be considered (domain 
S). Finally, each study was classified as having a high risk of bias, some concerns or a low risk of bias.

Both the PEDro and RoB2 tools were applied by two independent researchers, with any disagreement resolved through consensus.

\subsection{Data Extraction}

Data from each individual study were collected for every variable presented in Table 1 , including: (1) the first author, year and country; (2) the number and characteristics of participants and the sports modality; (3) the participants' daily caffeine intake; (4) the menstrual cycle phase and the presence of women using oral contraceptives; (5) the caffeine administration form, timing and dosage; (6) the state of fatigue when the athletes were tested (rest/fatigue condition); (7) the main performance outcomes.

For item 5, if the experiment involved different conditions besides isolated caffeine (e.g., mixing sodium phosphate with caffeine [26]), we only included the results of the isolated caffeine condition [26,27]. Regarding item 6 , we identified three possible conditions: (a) fatigue: tests developed after a fatigue-inducing protocol, match or strenuous effort that would cause fatigue to the participant; (b) match or simulated match: efforts developed during a regular match situation with official rules (in some cases the match duration was modified); (c) rest: tests developed without previous fatigue. These conditions were analyzed separately, given that the effects of caffeine might be different in rested and fatigued states. In a rested condition, the aim of evaluating caffeine intake would be to assess its effect on the performance of a specific task (e.g., jump performance). However, in the fatigued condition, the main aim would be to evaluate the effect of caffeine intake in minimizing the performance decline associated with fatigue by modulating the fatigue itself or its perception (e.g., jump performance after a soccer match or at half-time). Finally, for the match variables, the main aim would be to evaluate the effects of caffeine ingestion during real match situations (accelerations, decelerations, etc.) that are influenced by both physical and cognitive factors. For item 7 , the main outcomes selected were team-sport performance variables such as: jump performance; single sprint and RSA performance; agility tests; maximal voluntary isometric-, concentric- and eccentric-force tests; muscular endurance; anaerobic power (Wingate test); specific task performance (e.g., throwing a ball of the specific sport); specific match variables (body impacts, sprint speed, total sprint distance, accelerations and decelerations). We also considered the rating of perceived exertion (RPE) and fatigue indexes, as they are reliable proxies for physical performance despite not being direct outcomes of athletic performance.

\subsection{Meta-Analyses}

For the meta-analyses, we collected mean and error measures or effect sizes with confidence intervals. When these were not provided or when mean and error measures were only presented in figures, we contacted the corresponding authors [27-34] to obtain specific information (all authors replied).

Five studies included both males and females and analyzed them together, presenting pooled data. We collected mean and error values only for the female group after contacting the corresponding authors of two of the studies [14,35]. We did not include two studies $[36,37]$ that presented both sexes because the corresponding authors confirmed that they involved the same participants and tests that were presented in the following two studies that were included in the meta-analysis [32,38]. We did not contact the authors of one study that analyzed males and females together [13] because the measured main outcomes of the study were not of interest for the present review and meta-analysis. 
Table 1. Characteristics of studies included in the systematic review.

\begin{tabular}{|c|c|c|c|c|c|c|c|}
\hline $\begin{array}{c}\text { Authors, Year, } \\
\text { (Country) and } \\
\text { PEDro Score }\end{array}$ & $\begin{array}{l}\text { Sample } \\
\text { Level }^{+}\end{array}$ & $\begin{array}{c}\text { Caffeine Consumption or } \\
\text { Restrictions }\end{array}$ & $\begin{array}{l}\text { Menstrual Cycle and } \\
\text { Oral Contraceptives }\end{array}$ & $\begin{array}{c}\text { Timing }+ \\
\text { Intervention }+ \text { Washout }\end{array}$ & $\begin{array}{l}\text { Sample } \\
\text { State }\end{array}$ & Outcomes & $\mathbf{R}$ \\
\hline \multirow{3}{*}{$\begin{array}{l}\text { Astorino et al. } \\
2011 \\
\text { (USA) } \\
\text { PEDro: } 8 / 10\end{array}$} & \multirow{3}{*}{$\begin{array}{l}15 \text { NAIA soccer } \\
\text { players (19.5 } \pm 1.1 \\
\text { years) Level: } \\
\text { semi-professional }\end{array}$} & \multirow{3}{*}{$\begin{array}{l}\text { 12/15 were caffeine consumers } \\
\text { (dose not reported) } \\
\text { Instructed not to ingest any } \\
\text { caffeine } 48 \mathrm{~h} \text { before each trial }\end{array}$} & \multirow{3}{*}{ Not controlled } & \multirow{3}{*}{$\begin{array}{c}60 \mathrm{~min} \text { pre-test } \\
\text { CAF: Red bull (80 mg: } 1.3 \mathrm{mg} / \mathrm{kg}) \\
\text { PLA: Canada dry ginger ale Washout: } \\
72-96 \mathrm{~h}\end{array}$} & Rest & Agility $t$-test: Set $1 / 3$ of 8 reps. & $\rightleftarrows$ \\
\hline & & & & & \multirow{2}{*}{ Fatigue } & Agility $t$-test: Sets 2 and $3 / 3$ of 8 reps & $\rightleftarrows$ \\
\hline & & & & & & RPE & $\rightleftarrows$ \\
\hline \multirow{8}{*}{$\begin{array}{c}\text { Del Coso et al. } \\
2013 \text { (Spain) } \\
\text { PEDro: } 10 / 10\end{array}$} & \multirow{8}{*}{$\begin{array}{l}16 \text { rugby sevens } \\
\text { National Team } \\
\text { (23 } \pm 2 \text { years)Level: } \\
\text { elite }\end{array}$} & \multirow{8}{*}{$\begin{array}{l}\text { Light caffeine consumers: }<60 \\
\text { mg/day } \\
\text { Encouraged to abstain from all } \\
\text { dietary sources of caffeine for } 48 \mathrm{~h} \\
\text { before }\end{array}$} & \multirow{8}{*}{ Not controlled } & \multirow{8}{*}{$\begin{array}{c}60 \text { min pre-test } \\
\text { CAF: Powder caffeine-energy drink } 3 \\
\mathrm{mg} / \mathrm{kg}\left(\text { Fure }^{\circledR}\right) \\
\text { PLA: Powder drink } 0 \mathrm{mg} / \mathrm{kg} \\
\text { Washout: } 72 \mathrm{~h}\end{array}$} & Rest & $6 \times 30 \mathrm{~m}$ sprint test & $\rightleftarrows$ \\
\hline & & & & & \multirow{6}{*}{ Match } & Distance covered walking & $\rightleftarrows$ \\
\hline & & & & & & Distance covered cruising & $\uparrow$ \\
\hline & & & & & & Distance covered striding & $\uparrow$ \\
\hline & & & & & & Distance covered high intensity running & $\uparrow$ \\
\hline & & & & & & Distance covered sprinting & $\uparrow$ \\
\hline & & & & & & Match: RPE & $\rightleftarrows$ \\
\hline & & & & & Fatigue & 15 s maximal CMJs: total power & $\uparrow$ \\
\hline \multirow{7}{*}{$\begin{array}{l}\text { Lee et al. } 2014 \\
\text { (Taiwan) } \\
\text { PEDro: } 10 / 10\end{array}$} & \multirow{7}{*}{$\begin{array}{c}11 \text { Division I } \\
\text { collegiate team-sport } \\
\text { athletes (Basketball or } \\
\text { Volleyball) } \\
\text { (21.3 } \pm 1.2 \text { years) } \\
\text { Level: } \\
\text { semi-professional }\end{array}$} & \multirow{7}{*}{$\begin{array}{l}\text { Light caffeine consumers: } \\
\quad 50-100 \mathrm{mg} / \text { day }\end{array}$} & \multirow{7}{*}{ Not controlled } & \multirow{7}{*}{$\begin{array}{c}60 \text { min pre-test } \\
\text { CAF: } 6 \mathrm{mg} / \mathrm{kg} \text { capsulesPLA: Cellulose } \\
\text { capsules } \\
\text { Washout: at least } 1 \text { week }\end{array}$} & Rest & Agility $t$-test & $\rightleftarrows$ \\
\hline & & & & & \multirow{6}{*}{ Fatigue } & Cycle-ergometer repeated sprint mean power & $\rightleftarrows$ \\
\hline & & & & & & Cycle-ergometer repeated sprint total work & $\rightleftarrows$ \\
\hline & & & & & & Cycle-ergometer repeated sprint decrement & $\rightleftarrows$ \\
\hline & & & & & & Agility $t$-test & $\rightleftarrows$ \\
\hline & & & & & & Blood lactate & $\downarrow$ \\
\hline & & & & & & RPE & $\rightleftarrows$ \\
\hline
\end{tabular}


Table 1. Cont.

\begin{tabular}{|c|c|c|c|c|c|c|c|}
\hline $\begin{array}{l}\text { Authors, Year, } \\
\text { (Country) and } \\
\text { PEDro Score }\end{array}$ & $\begin{array}{l}\text { Sample } \\
\text { Level }^{+}\end{array}$ & $\begin{array}{c}\text { Caffeine Consumption or } \\
\text { Restrictions }\end{array}$ & $\begin{array}{l}\text { Menstrual Cycle and } \\
\text { Oral Contraceptives }\end{array}$ & $\begin{array}{c}\text { Timing }+ \\
\text { Intervention }+ \text { Washout }\end{array}$ & $\begin{array}{l}\text { Sample } \\
\text { State }\end{array}$ & Outcomes & $\mathbf{R}$ \\
\hline \multirow{14}{*}{$\begin{array}{l}\text { Lara et al. } 2014 \\
\quad \text { (Spain) } \\
\text { PEDro: } 10 / 10\end{array}$} & \multirow{14}{*}{$\begin{array}{l}18 \text { soccer players ( } 21 \\
\pm 2 \text { years) Level: not } \\
\text { reported }\end{array}$} & \multirow{14}{*}{$\begin{array}{l}\text { Light caffeine consumers: not } \\
\text { more than one cup of coffee or } \\
\text { energy drink per day } \\
\text { Encouraged to abstain from all } \\
\text { dietary sources of caffeine for } 48 \mathrm{~h} \\
\text { before }\end{array}$} & \multirow{14}{*}{ Not controlled } & \multirow{14}{*}{$\begin{array}{c}60 \text { min pre-test } \\
\text { CAF: Powder caffeine-energy drink } 3 \\
\text { mg/kg (Fure }{ }^{\circledR} \text { ) } \\
\text { PLA: Powder drink } 0 \mathrm{mg} / \mathrm{kg} \\
\text { Washout: } 1 \text { week }\end{array}$} & \multirow{4}{*}{ Rest } & $7 \times 30 \mathrm{~m}$ sprint average speed & $\uparrow$ \\
\hline & & & & & & $7 \times 30 \mathrm{~m}$ sprint maximal speed & $\uparrow$ \\
\hline & & & & & & CMJ height & $\uparrow$ \\
\hline & & & & & & CMJ Power & $\rightleftarrows$ \\
\hline & & & & & \multirow{10}{*}{ Match } & Total distance covered & $\uparrow$ \\
\hline & & & & & & Time standing & $\uparrow$ \\
\hline & & & & & & Time walking & $\rightleftarrows$ \\
\hline & & & & & & Time running $(3.1-8 \mathrm{~km} / \mathrm{h})$ & $\uparrow$ \\
\hline & & & & & & Time running $(8.1-13 \mathrm{~km} / \mathrm{h})$ & $\uparrow$ \\
\hline & & & & & & Time running $(13.1-18$ km/h) & $\rightleftarrows$ \\
\hline & & & & & & Time running $(>18 \mathrm{~km} / \mathrm{h})$ & $\uparrow$ \\
\hline & & & & & & Number of sprint bouts & $\uparrow$ \\
\hline & & & & & & Maximal speed & $\rightleftarrows$ \\
\hline & & & & & & RPE & $\rightleftarrows$ \\
\hline \multirow{11}{*}{$\begin{array}{l}\text { Buck et al. } 2015 \\
\text { (Australia) } \\
\text { PEDro: } 10 / 10\end{array}$} & \multirow{11}{*}{$\begin{array}{l}12 \text { amateur } \\
\text { team-sports (netball, } \\
\text { basketball and soccer) } \\
(25.5 \pm 1.9 \text { years }) \\
\text { Level: amateur }\end{array}$} & \multirow{11}{*}{$\begin{array}{l}\text { Caffeine consumption not } \\
\text { reported Participants were } \\
\text { advised to abstain from } \\
\text { consuming CAF for } 48 \mathrm{~h} \text { prior to } \\
\text { each trial }\end{array}$} & \multirow{11}{*}{$\begin{array}{l}3 \text { days post (follicular } \\
\text { phase) menstruation } \\
9 \text { were taking Levlen } \\
\text { ED for birth control } \\
3 \text { took no oral } \\
\text { contraceptives }\end{array}$} & \multirow{11}{*}{$\begin{array}{c}60 \text { min pre-test } \\
\text { CAF: Capsule }(6 \mathrm{mg} / \mathrm{kg} \mathrm{BM}) \\
\text { PLA: Capsule } \\
(1 \mathrm{~g} \text { glucose }) \\
\text { Washout: } \approx 21 \text { days }\end{array}$} & \multirow{3}{*}{ Rest } & $6 \times 20 \mathrm{~m}$ sprint before PSM & $\rightleftarrows$ \\
\hline & & & & & & Best $6 \times 20 \mathrm{~m}$ sprint before PSM & $\rightleftarrows$ \\
\hline & & & & & & Total $6 \times 20 \mathrm{~m}$ sprint time before PSM & $\rightleftarrows$ \\
\hline & & & & & \multirow{8}{*}{ Fatigue } & $6 \times 20 \mathrm{~m}$ sprint half-time PSM & $\rightleftarrows$ \\
\hline & & & & & & $6 \times 20 \mathrm{~m}$ sprint after PSM & $\rightleftarrows$ \\
\hline & & & & & & Best $6 \times 20 \mathrm{~m}$ sprint half-time PSM & $\rightleftarrows$ \\
\hline & & & & & & Best $6 \times 20 \mathrm{~m}$ sprint after PSM & $\rightleftarrows$ \\
\hline & & & & & & Total $6 \times 20 \mathrm{~m}$ sprint time half-time PSM & $\rightleftarrows$ \\
\hline & & & & & & Total $6 \times 20 \mathrm{~m}$ sprint time after PSM & $\rightleftarrows$ \\
\hline & & & & & & RPE during and after PSM & $\rightleftarrows$ \\
\hline & & & & & & Blood lactate during and after PSM & $\rightleftarrows$ \\
\hline
\end{tabular}


Table 1. Cont.

\begin{tabular}{|c|c|c|c|c|c|c|c|}
\hline $\begin{array}{c}\text { Authors, Year, } \\
\text { (Country) and } \\
\text { PEDro Score }\end{array}$ & $\begin{array}{l}\text { Sample } \\
\text { Level }^{+}\end{array}$ & $\begin{array}{l}\text { Caffeine Consumption or } \\
\text { Restrictions }\end{array}$ & $\begin{array}{l}\text { Menstrual Cycle and } \\
\text { Oral Contraceptives }\end{array}$ & $\begin{array}{c}\text { Timing }+ \\
\text { Intervention }+ \text { Washout }\end{array}$ & $\begin{array}{l}\text { Sample } \\
\text { State }\end{array}$ & Outcomes & $\mathbf{R}$ \\
\hline \multirow{5}{*}{$\begin{array}{l}\text { Chen et al. } 2015 \\
\text { (Taiwan) } \\
\text { PEDro: } 10 / 10\end{array}$} & \multirow{5}{*}{$\begin{array}{c}10 \text { elite collegiate } \\
\text { athletes (tennis, } \\
\text { soccer, basketball) } \\
\text { (19.9 } \pm 0.9 \text { years) } \\
\text { Level: } \\
\text { semi-professional }\end{array}$} & \multirow{5}{*}{$\begin{array}{l}\text { No regular caffeine consumption }< \\
\qquad 200 \mathrm{mg} / \text { week }\end{array}$} & \multirow{5}{*}{$\begin{array}{l}\text { Instructed to } \\
\text { participate during } \\
\text { their early follicular } \\
\text { phase and avoid } \\
\text { taking contraception }\end{array}$} & \multirow{5}{*}{$\begin{array}{l}60 \mathrm{~min} \text { pre-test } \\
\text { CAF: Capsule } 6 \mathrm{mg} / \mathrm{kg} \\
\text { PLA: Diet flour in capsule } \\
\text { Washout: } 1 \text { week }\end{array}$} & \multirow[b]{2}{*}{ Rest } & MVIC & $\uparrow$ \\
\hline & & & & & & Isometric fatigue protocol & $\uparrow$ \\
\hline & & & & & \multirow{3}{*}{ Fatigue } & Fatigued MVIC & $\uparrow$ \\
\hline & & & & & & Fatigue index & $\uparrow$ \\
\hline & & & & & & Blood lactate & $\downarrow$ \\
\hline \multirow{5}{*}{$\begin{array}{l}\text { Mahdavi et al. } \\
2015 \\
\text { (Iran) PEDro: } \\
10 / 10\end{array}$} & \multirow{5}{*}{$\begin{array}{c}24 \text { basketball players } \\
(24.2 \pm 2.6 \text { years }) \\
\text { Level: not reported }\end{array}$} & \multirow{5}{*}{$116.8 \pm 26.7 \mathrm{mg} /$ day } & \multirow{5}{*}{ Not controlled } & \multirow{5}{*}{$\begin{array}{c}70 \mathrm{~min} \text { pre-test } \\
\text { CAF: Capsules } 5 \mathrm{mg} / \mathrm{kg} \\
\text { PLA: Capsules with dextrose Washout: } 1 \\
\text { week }\end{array}$} & \multirow{5}{*}{ Rest } & 30 s WT: Mean power & $\rightleftarrows$ \\
\hline & & & & & & 30 s WT: End power & $\rightleftarrows$ \\
\hline & & & & & & 30 s WT: Power drop & $\rightleftarrows$ \\
\hline & & & & & & 30 s WT: Fatigue index & $\rightleftarrows$ \\
\hline & & & & & & 30 s WT: Lactate & $\uparrow$ \\
\hline \multirow{6}{*}{$\begin{array}{l}\text { Fernandez- } \\
\text { Campos et al. } \\
2015 \text { (Costa } \\
\text { Rica) } \\
\text { PEDro: } 9 / 10\end{array}$} & \multirow{6}{*}{$\begin{array}{l}19 \text { volleyball players } \\
\text { from the elite league } \\
\text { of Costa Rica ( } 22.3 \pm \\
4.9 \text { years) Level: elite }\end{array}$} & \multirow{6}{*}{ Not reported } & \multirow{6}{*}{ Not controlled } & \multirow{6}{*}{$\begin{array}{c}30 \text { min pre-test } \\
\text { CAF: Energy drink } 6 \mathrm{ml} / \mathrm{kg} \text { with } 73 \mathrm{mg} \\
\text { of CAF in } 273 \mathrm{~mL} .(1.7 \mathrm{mg} / \mathrm{kg}) \\
\text { PLA: flavored drink } \\
\text { Washout: } 1 \text { week }\end{array}$} & \multirow{6}{*}{ Rest } & Right handgrip strength & $\uparrow$ \\
\hline & & & & & & Left handgrip strength & $\rightleftarrows$ \\
\hline & & & & & & SJ height & $\rightleftarrows$ \\
\hline & & & & & & WT peak power & $\rightleftarrows$ \\
\hline & & & & & & WT mean power & $\rightleftarrows$ \\
\hline & & & & & & WT fatigue index & $\rightleftarrows$ \\
\hline
\end{tabular}


Table 1. Cont.

\begin{tabular}{|c|c|c|c|c|c|c|c|}
\hline $\begin{array}{l}\text { Authors, Year, } \\
\text { (Country) and } \\
\text { PEDro Score }\end{array}$ & $\begin{array}{l}\text { Sample } \\
\text { Level }^{+}\end{array}$ & $\begin{array}{l}\text { Caffeine Consumption or } \\
\text { Restrictions }\end{array}$ & $\begin{array}{l}\text { Menstrual Cycle and } \\
\text { Oral Contraceptives }\end{array}$ & $\begin{array}{c}\text { Timing }+ \\
\text { Intervention }+ \text { Washout }\end{array}$ & $\begin{array}{l}\text { Sample } \\
\text { State }\end{array}$ & Outcomes & $\mathbf{R}$ \\
\hline \multirow{18}{*}{$\begin{array}{l}\text { Perez-Lopez } \\
\text { et al. } 2015 \\
\text { (Spain) } \\
\text { PEDro: } 10 / 10\end{array}$} & \multirow{18}{*}{$\begin{array}{l}13 \text { volleyball players } \\
\text { from the second } \\
\text { division of the } \\
\text { Spanish league ( } 25.2 \\
\pm 4.8) \\
\text { Level: } \\
\text { semi-professional }\end{array}$} & \multirow{18}{*}{$\begin{array}{l}\text { On the day of the trial participants } \\
\text { were encouraged to refrain from } \\
\text { all dietary sources of caffeine }\end{array}$} & \multirow{18}{*}{$\begin{array}{l}4 \text { during follicular } \\
\text { phase9 during luteal } \\
\text { phase }\end{array}$} & \multirow{18}{*}{$\begin{array}{c}60 \text { min pre-test } \\
\text { CAF: Powder energy drink }\left(\text { Fure }^{\circledR}\right) 3 \\
\text { mg } / \mathrm{kg} \\
\text { PLA: Powder with } 0 \mathrm{mg} / \mathrm{kg} \text { of CAF } \\
\text { Washout: } 1 \text { week }\end{array}$} & \multirow{8}{*}{ Rest } & Handgrip & $\uparrow$ \\
\hline & & & & & & Spike jump height and peak power & $\uparrow$ \\
\hline & & & & & & Block jump height and peak power & $\uparrow$ \\
\hline & & & & & & Squat jump height and peak power & $\uparrow$ \\
\hline & & & & & & CMJ height and peak power & $\uparrow$ \\
\hline & & & & & & Agility t-test & $\uparrow$ \\
\hline & & & & & & Standing spike ball velocity & $\uparrow$ \\
\hline & & & & & & Jumping spike ball velocity & $\uparrow$ \\
\hline & & & & & \multirow{10}{*}{ Match } & Body accelerations & $\uparrow$ \\
\hline & & & & & & Positive game actions & $\uparrow$ \\
\hline & & & & & & Neutral game actions & $\rightleftarrows$ \\
\hline & & & & & & Negative game actions & $\uparrow$ \\
\hline & & & & & & Body impacts $0-1 \mathrm{~g}$ & $\uparrow$ \\
\hline & & & & & & Body impacts $1.1-2 \mathrm{~g}$ & $\uparrow$ \\
\hline & & & & & & Body impacts $2.1-3 \mathrm{~g}$ & $\uparrow$ \\
\hline & & & & & & Body impacts $3.1-4 \mathrm{~g}$ & $\rightleftarrows$ \\
\hline & & & & & & Body impacts $4.1-5 \mathrm{~g}$ & $\uparrow$ \\
\hline & & & & & & Body impacts 5.1-6 g & $\uparrow$ \\
\hline
\end{tabular}


Table 1. Cont

\begin{tabular}{|c|c|c|c|c|c|c|c|}
\hline $\begin{array}{l}\text { Authors, Year, } \\
\text { (Country) and } \\
\text { PEDro Score }\end{array}$ & $\begin{array}{l}\text { Sample } \\
\text { Level }^{+}\end{array}$ & $\begin{array}{l}\text { Caffeine Consumption or } \\
\text { Restrictions }\end{array}$ & $\begin{array}{l}\text { Menstrual Cycle and } \\
\text { Oral Contraceptives }\end{array}$ & $\begin{array}{c}\text { Timing }+ \\
\text { Intervention }+ \text { Washout }\end{array}$ & $\begin{array}{l}\text { Sample } \\
\text { State }\end{array}$ & Outcomes & $\mathbf{R}$ \\
\hline \multirow{25}{*}{$\begin{array}{l}\text { Ali et al. 2016a } \\
\text { (New Zealand) } \\
\text { PEDro: } 10 / 10\end{array}$} & \multirow{25}{*}{$\begin{array}{l}10 \text { healthy team sport } \\
\text { players (soccer, } \\
\text { hockey and netball) } \\
(24 \pm 4 \text { years }) \\
\text { Level: amateur and } \\
\text { elite }\end{array}$} & \multirow{25}{*}{$\begin{array}{l}\text { Self-reported daily caffeine intake } \\
\text { varied from } 0 \text { to } 300 \mathrm{mg} / \text { day }\end{array}$} & \multirow{25}{*}{$\begin{array}{c}\text { All participants were } \\
\text { taking a monophasic } \\
\text { oral } \\
\text { contraceptive } \\
\text { (Monofeme, } \\
\text { Microgynon, Levlen } \\
\text { ED or Nordette) }\end{array}$} & \multirow{25}{*}{$\begin{array}{c}60 \mathrm{~min} \text { pre-test } \\
\text { CAF: Capsules } 6 \mathrm{mg} / \mathrm{kg} \\
\text { PLA: Capsules with artificial sweetener } \\
\text { Washout: } 13-17 \text { days }\end{array}$} & \multirow{6}{*}{ Rest } & Knee flexor ecc. PT pre-PSM & $\rightleftarrows$ \\
\hline & & & & & & Knee extensor ecc. PT pre-PSM & $\rightleftarrows$ \\
\hline & & & & & & Knee flexor ecc. Power pre-PSM & $\rightleftarrows$ \\
\hline & & & & & & Knee extensor ecc. Power pre-PSM & $\rightleftarrows$ \\
\hline & & & & & & Isometric knee flexor pre-PSM & $\rightleftarrows$ \\
\hline & & & & & & $\mathrm{CMJ}$ height and power pre-PSM & $\rightleftarrows$ \\
\hline & & & & & \multirow{19}{*}{ Fatigue } & Knee flexor ecc. PT mid-PSM & $\uparrow$ \\
\hline & & & & & & Knee flexor ecc. PT post-PSM & $\rightleftarrows$ \\
\hline & & & & & & Knee flexor ecc. PT 12 h-post-PSM & $\uparrow$ \\
\hline & & & & & & Knee extensor ecc. PT mid-PSM & $\rightleftarrows$ \\
\hline & & & & & & Knee extensor ecc. PT post-PSM & $\rightleftarrows$ \\
\hline & & & & & & Knee extensor ecc. PT 12 h-post-PSM & $\rightleftarrows$ \\
\hline & & & & & & Knee flexor ecc. Power post-PSM & $\rightleftarrows$ \\
\hline & & & & & & Knee flexor ecc. Power 12 h-post-PSM & $\uparrow$ \\
\hline & & & & & & Knee extensor ecc. Power mid-PSM & $\uparrow$ \\
\hline & & & & & & Knee extensor ecc. Power post-PSM & $\rightleftarrows$ \\
\hline & & & & & & Knee extensor ecc. Power 12 h-post-PSM & $\rightleftarrows$ \\
\hline & & & & & & Isometric knee flexor mid-PSM & $\rightleftarrows$ \\
\hline & & & & & & Isometric knee flexor post-PSM & $\rightleftarrows$ \\
\hline & & & & & & Isometric knee flexor $12 \mathrm{~h}$ post-PSM & $\rightleftarrows$ \\
\hline & & & & & & Isometric knee extensor mid-PSM & $\rightleftarrows$ \\
\hline & & & & & & Isometric knee extensor post-PSM & $\rightleftarrows$ \\
\hline & & & & & & Isometric knee extensor $12 \mathrm{~h}$ post-PSM & $\rightleftarrows$ \\
\hline & & & & & & CMJ height and power post-PSM & $\rightleftarrows$ \\
\hline & & & & & & CMJ height and power $12 \mathrm{~h}$ post-PSM & $\rightleftarrows$ \\
\hline
\end{tabular}


Table 1. Cont.

\begin{tabular}{|c|c|c|c|c|c|c|c|}
\hline $\begin{array}{l}\text { Authors, Year, } \\
\text { (Country) and } \\
\text { PEDro Score }\end{array}$ & $\begin{array}{l}\text { Sample } \\
\text { Level }^{+}\end{array}$ & $\begin{array}{l}\text { Caffeine Consumption or } \\
\text { Restrictions }\end{array}$ & $\begin{array}{l}\text { Menstrual Cycle and } \\
\text { Oral Contraceptives }\end{array}$ & $\begin{array}{c}\text { Timing }+ \\
\text { Intervention }+ \text { Washout }\end{array}$ & $\begin{array}{l}\text { Sample } \\
\text { State }\end{array}$ & Outcomes & $\mathbf{R}$ \\
\hline $\begin{array}{l}\text { Ali et al. 2016b } \\
\text { (New Zealand) } \\
\text { PEDro: } 10 / 10\end{array}$ & $\begin{array}{l}10 \text { healthy team sport } \\
\text { players (soccer, } \\
\text { hockey and netball) } \\
\text { (24 } \pm 4 \text { years) Level: } \\
\text { amateur and elite }\end{array}$ & $\begin{array}{l}\text { Self-reported daily caffeine intake } \\
\text { varied from } 0 \text { to } 300 \mathrm{mg} / \text { day }\end{array}$ & $\begin{array}{l}\text { All participants were } \\
\text { taking a monophasic } \\
\text { oralcontraceptive }\end{array}$ & $\begin{array}{c}60 \mathrm{~min} \text { pre-test } \\
\text { CAF: Capsules } 6 \mathrm{mg} / \mathrm{kg} \\
\text { PLA: Capsules with artificial sweetener } \\
\text { Washout: } 13-17 \text { days }\end{array}$ & Fatigue & RPE & $\rightleftarrows$ \\
\hline \multirow{8}{*}{$\begin{array}{l}\text { Portillo et al. } \\
2017 \\
\text { (Spain) } \\
\text { PEDro: } 10 / 10\end{array}$} & \multirow{8}{*}{$\begin{array}{l}16 \text { rugby sevens } \\
\text { national team players } \\
\text { (23 } \pm 2 \text { years) Level: } \\
\text { elite }\end{array}$} & \multirow{8}{*}{$\begin{array}{l}\text { Light caffeine consumers: }<60 \\
\text { mg/day }\end{array}$} & \multirow{8}{*}{ Not controlled } & \multirow{8}{*}{$\begin{array}{c}60 \text { min pre-test } \\
\text { CAF: Powder } 3 \mathrm{mg} / \mathrm{kg} \\
\text { PLA: Powder with } 0 \mathrm{mg} / \mathrm{kg} \text { of CAF } \\
\text { Washout: } 72 \mathrm{~h}\end{array}$} & \multirow{8}{*}{ Match } & Body impacts 0-6 g & $\uparrow$ \\
\hline & & & & & & Body impacts $6.01-6.5 \mathrm{~g}$ & $\uparrow$ \\
\hline & & & & & & Body impacts $6.51-7 \mathrm{~g}$ & $\uparrow$ \\
\hline & & & & & & Body impacts $7.01-8 \mathrm{~g}$ & $\rightleftarrows$ \\
\hline & & & & & & Body impacts $8.01-10 \mathrm{~g}$ & $\uparrow$ \\
\hline & & & & & & Body impacts $>10 \mathrm{~g}$ & $\rightleftarrows$ \\
\hline & & & & & & Frequency of technical action & $\rightleftarrows$ \\
\hline & & & & & & Ratings of skill performance & $\rightleftarrows$ \\
\hline \multirow{10}{*}{$\begin{array}{l}\text { Puente et al. } \\
2017 \\
\text { (Spain) } \\
\text { PEDro: } 10 / 10\end{array}$} & \multirow{10}{*}{$\begin{array}{c}10 \text { professional } \\
\text { basketaball players } \\
(27.9 \pm 6.1 \text { years }) \\
\text { Level: } \\
\text { semi-professional and } \\
\text { elite }\end{array}$} & \multirow{10}{*}{$\begin{array}{l}\text { Light caffeine consumers }<100 \\
\text { mg/day } \\
\text { Encouraged to abstain from CAF } \\
\text { ingestion during the study }\end{array}$} & \multirow{10}{*}{$\begin{array}{l}\text { All participants were } \\
\text { tested during their } \\
\text { luteal phase }\end{array}$} & \multirow{10}{*}{$\begin{array}{c}60 \mathrm{~min} \text { pre-test } \\
\text { CAF: Capsule } 3 \mathrm{mg} / \mathrm{kgPLA} \text { : Capsule } 0 \\
\mathrm{mg} / \mathrm{kg} \text { of CAF } \\
\text { Washout: } 1 \text { week }\end{array}$} & \multirow{3}{*}{ Rest } & Abalakov jump & \multirow{10}{*}{ NA } \\
\hline & & & & & & Free throws & \\
\hline & & & & & & CODAT with ball & \\
\hline & & & & & \multirow{7}{*}{ Match } & Body impacts $0-0.99 \mathrm{~g}$ & \\
\hline & & & & & & Body impacts $1-1.99 \mathrm{~g}$ & \\
\hline & & & & & & Body impacts $2-2.99 \mathrm{~g}$ & \\
\hline & & & & & & Body impacts 3-3.99g & \\
\hline & & & & & & Body impacts $4-4.99 \mathrm{~g}$ & \\
\hline & & & & & & Body impacts $>5 \mathrm{~g}$ & \\
\hline & & & & & & RPE & \\
\hline
\end{tabular}


Table 1. Cont.

\begin{tabular}{|c|c|c|c|c|c|c|c|}
\hline $\begin{array}{l}\text { Authors, Year, } \\
\text { (Country) and } \\
\text { PEDro Score }\end{array}$ & $\begin{array}{l}\text { Sample } \\
\text { Level }^{+}\end{array}$ & $\begin{array}{c}\text { Caffeine Consumption or } \\
\text { Restrictions }\end{array}$ & $\begin{array}{l}\text { Menstrual Cycle and } \\
\text { Oral Contraceptives }\end{array}$ & $\begin{array}{c}\text { Timing }+ \\
\text { Intervention }+ \text { Washout }\end{array}$ & $\begin{array}{l}\text { Sample } \\
\text { State }\end{array}$ & Outcomes & $\mathbf{R}$ \\
\hline \multirow{3}{*}{$\begin{array}{l}\text { Pfeifer et al. } \\
2017 \\
\text { (USA) } \\
\text { PEDro: } 8 / 10\end{array}$} & \multirow{3}{*}{$\begin{array}{l}8 \text { volleyball NAIA } \\
\text { volleyball } \\
\text { (18-22 years)Level: } \\
\text { semi-professional }\end{array}$} & \multirow[t]{3}{*}{$\begin{array}{l}\text { CAF consumption was not } \\
\text { restricted }\end{array}$} & \multirow[t]{3}{*}{ Not controlled } & \multirow{3}{*}{$\begin{array}{c}\text { Prior to and during the competition } \\
\text { CAF: } \\
\text { PowerBar }{ }^{\circledR} \text { PowerGel }{ }^{\circledR} \\
50 \mathrm{mg} \text { of caffeine. Averaged } 1.39 \mathrm{mg} / \mathrm{kg} \\
\text { PLA: Non-nutritive gel } \\
\text { Washout: } \approx 1 \text { week }\end{array}$} & \multirow[t]{3}{*}{ Fatigue } & Vertical jump with a two-step approach & $\rightleftarrows$ \\
\hline & & & & & & Three cone drill agility & $\rightleftarrows$ \\
\hline & & & & & & $6 \times 30 \mathrm{~m}$ sprint & $\rightleftarrows$ \\
\hline \multirow{10}{*}{$\begin{array}{l}\text { Stojanovic et al. } \\
2019 \\
\text { (Serbia) } \\
\text { PEDro: } 10 / 10\end{array}$} & \multirow{10}{*}{$\begin{array}{c}10 \text { professional } \\
\text { basketball players } \\
(20.2 \pm 3.9 \text { years }) \\
\text { Level: elite }\end{array}$} & \multirow{10}{*}{$\begin{array}{l}\text { Light caffeine consumers: }<100 \\
\text { mg/day }\end{array}$} & \multirow{10}{*}{$\begin{array}{l}\text { Completed testing in } \\
\text { the luteal phase of } \\
\text { their menstrual cycle } \\
\text { Use of oral } \\
\text { contraceptives not } \\
\text { reported by authors }\end{array}$} & \multirow{10}{*}{$\begin{array}{c}60 \text { min pre-test } \\
\text { CAF: Capsule ( } 3 \text { mg/kg BM) } \\
\text { PLA: Capsule (Dextrose) } \\
\text { Washout: } 1 \text { week }\end{array}$} & \multirow{10}{*}{ Rest } & SJ height & $\rightleftarrows$ \\
\hline & & & & & & ABA height & $\rightleftarrows$ \\
\hline & & & & & & Lane agility & $\rightleftarrows$ \\
\hline & & & & & & $5 \mathrm{~m}$ sprint & $\rightleftarrows$ \\
\hline & & & & & & $10 \mathrm{~m}$ sprint & $\uparrow$ \\
\hline & & & & & & $20 \mathrm{~m}$ sprint & $\uparrow$ \\
\hline & & & & & & $\begin{array}{l}5 \mathrm{~m} \text { sprint-dibbling } \\
10 \mathrm{~m} \text { sprint-dibbling }\end{array}$ & $\underset{\rightleftarrows}{\rightleftarrows}$ \\
\hline & & & & & & $20 \mathrm{~m}$ sprint-dibbling & $\rightleftarrows$ \\
\hline & & & & & & RSP: Suicide run & $\rightleftarrows$ \\
\hline & & & & & & RPE & $\uparrow$ \\
\hline \multirow{2}{*}{$\begin{array}{c}\text { Tan et al. } 2020 \\
\text { (Singapore) } \\
\text { PEDro: } 8 / 10 \\
\end{array}$} & \multirow{2}{*}{$\begin{array}{l}6 \text { basketball players } \\
\text { Level: } \\
\text { semi-professional } \\
\end{array}$} & \multirow[t]{2}{*}{ Less than $200 \mathrm{mg}$ caffeine per day } & \multirow[t]{2}{*}{ Not controlled } & \multirow{2}{*}{$\begin{array}{c}60 \mathrm{~min} \text { pre-test } \\
\text { CAF: Powders (6 mg/kg BM) } \\
\text { PLA: Powders (Maltodextrine) } \\
\text { Washout: } 72 \mathrm{~h} \\
\end{array}$} & \multirow[t]{2}{*}{ Fatigue } & Free throws & \multirow{2}{*}{ NA } \\
\hline & & & & & & RPE & \\
\hline
\end{tabular}


Table 1. Cont.

\begin{tabular}{|c|c|c|c|c|c|c|c|}
\hline $\begin{array}{l}\text { Authors, Year, } \\
\text { (Country) and } \\
\text { PEDro Score }\end{array}$ & $\begin{array}{l}\text { Sample } \\
\text { Level }^{+}\end{array}$ & $\begin{array}{l}\text { Caffeine Consumption or } \\
\text { Restrictions }\end{array}$ & $\begin{array}{l}\text { Menstrual Cycle and } \\
\text { Oral Contraceptives }\end{array}$ & $\begin{array}{c}\text { Timing }+ \\
\text { Intervention }+ \text { Washout }\end{array}$ & $\begin{array}{l}\text { Sample } \\
\text { State }\end{array}$ & Outcomes & $\mathbf{R}$ \\
\hline \multirow{15}{*}{$\begin{array}{l}\text { Muñoz et al. } \\
2020 \\
\text { (Spain) } \\
\text { PEDro: } 10 / 10\end{array}$} & \multirow{15}{*}{$\begin{array}{c}15 \text { elite handball } \\
\text { players }(22.6 \pm 3.6 \\
\text { years }) \\
\text { Level: elite }\end{array}$} & \multirow{15}{*}{$\begin{array}{l}\text { Light caffeine consumers: } 50 \pm 30 \\
\text { mg/day }\end{array}$} & \multirow{15}{*}{$\begin{array}{l}10 \text { during follicular } \\
\text { phase } \\
5 \text { during luteal phase } \\
\text { Use of oral } \\
\text { contraceptives not } \\
\text { reported by authors }\end{array}$} & \multirow{15}{*}{$\begin{array}{c}60 \text { min pre-test } \\
\text { CAF: Capsule ( } 3 \text { mg/kg BM) } \\
\text { PLA: Capsule (Cellulose) } \\
\text { Washout: } 1 \text { week }\end{array}$} & \multirow{8}{*}{ Rest } & $7 \mathrm{~m}$ ball throws & $\uparrow$ \\
\hline & & & & & & $9 \mathrm{~m}$ ball throws & $\uparrow$ \\
\hline & & & & & & $7 \mathrm{~m}$ ball throws goalk. & $\uparrow$ \\
\hline & & & & & & 9m ball throws goalk. & $\uparrow$ \\
\hline & & & & & & CMJ height & $\uparrow$ \\
\hline & & & & & & Handgrip & $\uparrow$ \\
\hline & & & & & & Agility: MATT & $\rightleftarrows$ \\
\hline & & & & & & $30 \mathrm{~m}$ sprint & $\uparrow$ \\
\hline & & & & & \multirow{7}{*}{ Match } & Accelerations frequency & $\uparrow$ \\
\hline & & & & & & Decelerations frequency & $\uparrow$ \\
\hline & & & & & & Body impacts & $\uparrow$ \\
\hline & & & & & & Total distance & $\rightleftarrows$ \\
\hline & & & & & & Sprint distance & $\rightleftarrows$ \\
\hline & & & & & & Maximal speed & $\rightleftarrows$ \\
\hline & & & & & & RPE & $\rightleftarrows$ \\
\hline
\end{tabular}


Table 1. Cont.

\begin{tabular}{|c|c|c|c|c|c|c|c|}
\hline $\begin{array}{l}\text { Authors, Year, } \\
\text { (Country) and } \\
\text { PEDro Score }\end{array}$ & $\begin{array}{l}\text { Sample } \\
\text { Level }^{+}\end{array}$ & $\begin{array}{l}\text { Caffeine Consumption or } \\
\text { Restrictions }\end{array}$ & $\begin{array}{l}\text { Menstrual Cycle and } \\
\text { Oral Contraceptives }\end{array}$ & $\begin{array}{c}\text { Timing }+ \\
\text { Intervention }+ \text { Washout }\end{array}$ & $\begin{array}{l}\text { Sample } \\
\text { State }\end{array}$ & Outcomes & $\mathbf{R}$ \\
\hline \multirow[t]{2}{*}{$\begin{array}{l}\text { Karayigit et al. } \\
2021 \\
\text { (Turkey) } \\
\text { PEDro: } 10 / 10\end{array}$} & \multirow[t]{2}{*}{$\begin{array}{c}17 \text { female team sports } \\
\text { (rugby, handball and } \\
\text { soccer) } \\
23 \pm 2 \text { years) } \\
\text { Level: elite and } \\
\text { semi-professional }\end{array}$} & \multirow[t]{2}{*}{$\begin{array}{l}\text { Light caffeine consumers: } \\
<25 \mathrm{mg} / \text { day }\end{array}$} & \multirow[t]{2}{*}{$\begin{array}{l}\text { All sessions were } \\
\text { performed during the } \\
\text { luteal phase of the } \\
\text { menstrual cycle } \\
\text { All subjects stopped } \\
\text { oral contraceptive } \\
\text { consumpletion } 3 \\
\text { months before the } \\
\text { commencement of the } \\
\text { study }\end{array}$} & \multirow[t]{2}{*}{$\begin{array}{c}60 \mathrm{~min} \text { pre-test } \\
\text { CAF: Coffee (3 mg/kg BM) } \\
(6 \mathrm{mg} / \mathrm{kg} \mathrm{BM}) \\
\text { PLA: Decaffeinated coffee } \\
\text { Washout: } 48-72 \mathrm{~h}\end{array}$} & \multirow[t]{2}{*}{ Rest } & $\begin{array}{c}3 \text { set of repetitions to failure } 40 \% 1 \mathrm{RM} \text { bench } \\
\text { press }\end{array}$ & $\uparrow$ \\
\hline & & & & & & 3 set of repetitions to failure $40 \% 1 \mathrm{RM}$ squat & $\rightleftarrows$ \\
\hline
\end{tabular}

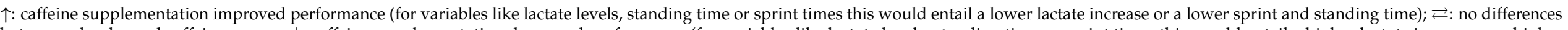

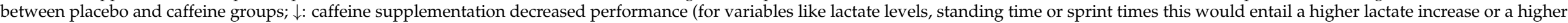

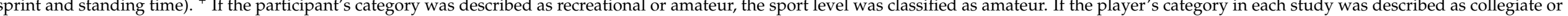

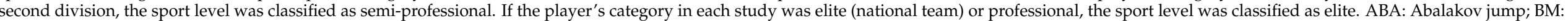

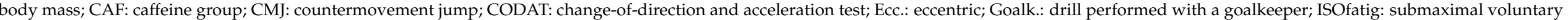

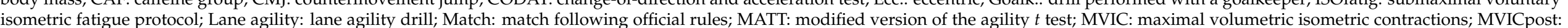

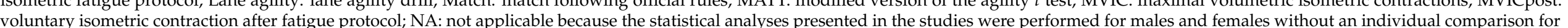

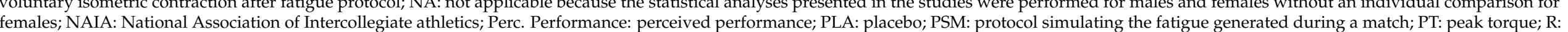
results; RPE: rate of perceived exertion; RSP: repeated sprint performance; SF: sodium phosphate; SJ: squat jump; SM: simulated match; SP: self-perceived; WT: Wingate test. 


\subsection{Statistical Analyses}

After calculating the standardized mean difference as the individual effect size of each study for each relevant variable, the results were pooled using the DerSimonian-Laird method in a random-effects meta-analysis [39]. A minimum of three studies was required in order to perform the meta-analyses. When calculating the standardized mean difference between conditions within each meta-analysis, the data were set to indicate that a positive value always represented a difference in performance favoring the caffeine condition.

A sensitivity analysis was performed excluding those studies that administered less than $2 \mathrm{mg}$ of caffeine per $\mathrm{kg}$ of body mass $[33,40,41]$ as this has been suggested to be the minimum effective ergogenic dose [17].

We tested the heterogeneity using the $\mathrm{I}^{2}$ statistic [42]. This statistic describes the variance between studies as a proportion of the total variance. A value of $25-50 \%$ indicates low heterogeneity, between $50-75 \%$ indicates moderate heterogeneity and $>75 \%$ indicates high heterogeneity.

\section{Results}

\subsection{Main Search}

The literature search provided a total of 588 studies, with 4 additional studies found through cross-referencing. A total of 54 full-text articles were read, and 18 met the inclusion criteria and were included in the systematic review. Figure 1 presents the PRISMA flow chart and the reasons for excluding articles from the final sample of selected studies.

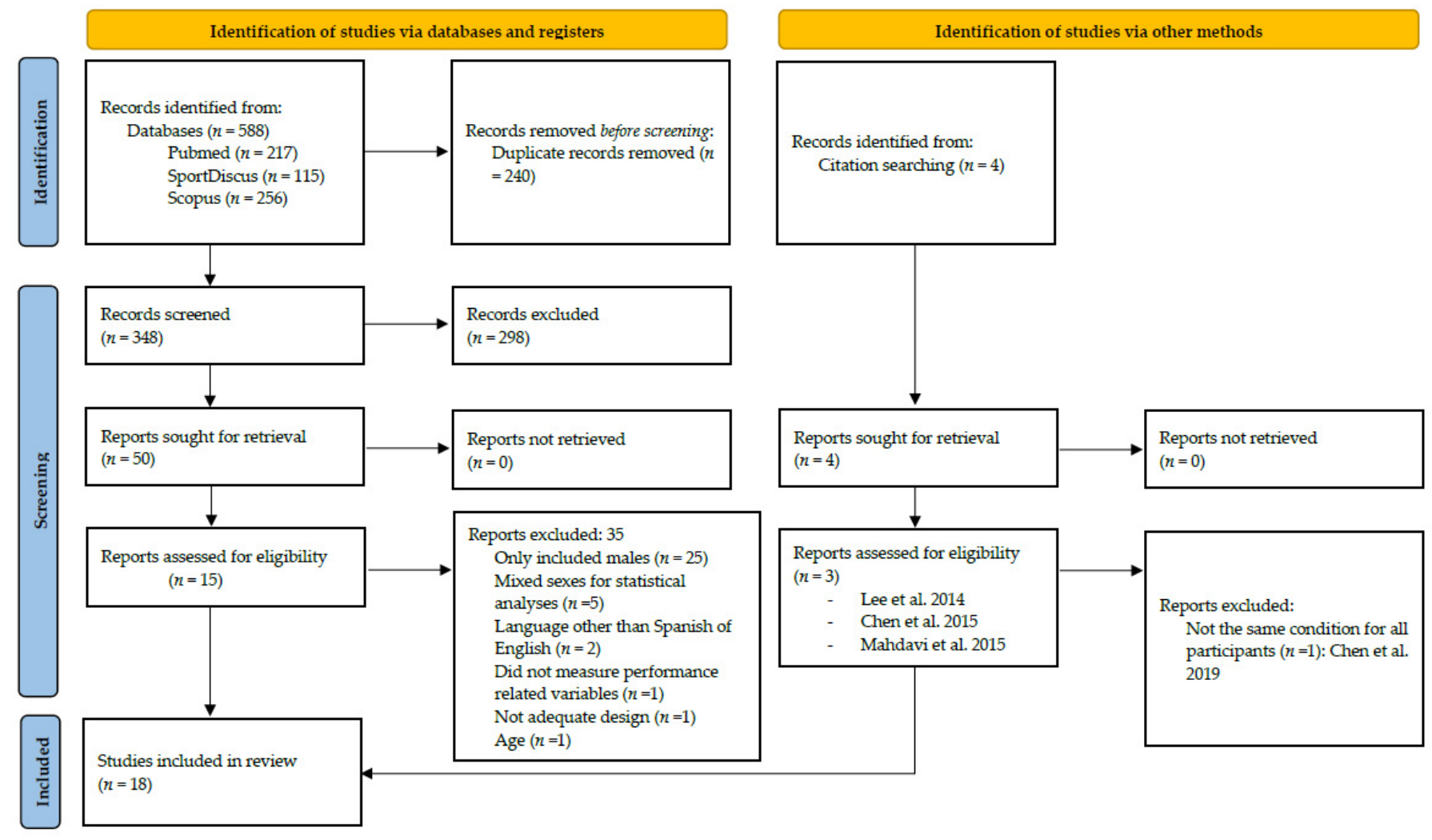

Figure 1. PRISMA flow-chart diagram.

\subsection{Quality Assessment and Risk of Bias}

The individual PEDro quality scores ranged from 8 to 10, being excellent in 15 studies and good in 3 studies (Supplementary Material: Table S2). Three crossover trials did not meet the requirements related to therapist and assessor blinding [33,35,40], and the study performed by Fernandez-Campos et al. [41] did not include drop-outs in the analysis. 
Regarding the RoB 2 tool results, 14 studies showed a low risk of bias in all domains, 4 studies demonstrated some concerns for domain 2 "bias due to deviations from intended interventions" and 3 studies showed some concerns for domain 4 "bias in measurement of the outcome" (Supplementary Material: Table S3). Additionally, all studies had a low risk of bias in the specific domain for crossover designs "bias arising from the period effect and carryover effect" (domain S). Thus, the overall biases were low for 14 studies, with some concern in the other studies.

\subsection{Description of Participants and Studies}

Six studies were performed in Spain. Two studies were developed in the United States of America, New Zealand and Taiwan. Australia, Iran, Costa Rica, Serbia, Turkey and Singapore each provided one study. The origin of each individual study is presented in the first column of Table 1.

The main characteristics of each study are presented in Table 1 . The 18 studies included provided a total of 240 young adult female TSA (the mean age for all studies was in the range of 18 to 26 years). Of this sample, 50 participants were basketball players $[14,35,38,43], 40$ were volleyball players [30,33,41], 37 were soccer players [29,40], 32 were rugby players [28,31] and 15 were handball players [32]. The rest of the studies used a sample of mixed TSA including basketball, volleyball, handball, soccer, rugby, softball, hockey and netball players [26,27,34,44-46].

\subsection{Caffeine Supplementation and Doses}

Caffeine doses ranged from $1.3 \mathrm{mg} / \mathrm{kg}$ to $6 \mathrm{mg} / \mathrm{kg}$, mainly ingested through: capsules: nine studies [14,26,27,32,34,38,43-45], powders: five studies [28-31,35], energy drinks: two studies $[40,41]$, power bars: one study [33] or coffee [46].

All studies supplied the caffeine 60 min before the experiments, except for Mahdavi et al. [43], who provided the caffeine supplementation 70 min before, Fernandez-Campos et al. [41] who provided it 30 min before and Pfeifer et al. [33] who specified that the dose "was administered immediately prior to and during the competition".

Regarding caffeine withdrawal as part of the standardization procedures, although different instructions to volunteers were found among the studies (Specified in Table 1), most studies required participants to abstain from all dietary sources of caffeine for $48 \mathrm{~h}$ before the trials.

Finally, regarding the days that passed between the placebo and caffeine conditions, five studies performed washout periods of 48 to $96 \mathrm{~h}$, with most studies performing oneweek washout periods (10 studies). Three studies performed even longer washouts (13 to 21 days). Individual information for each study is provided in Table 1.

\subsection{Menstrual Cycle}

In the first four studies that were published between 2011 and 2014 [27-29,40], the phase of the menstrual cycle and the use of oral contraceptives was not reported. From 2015, some studies reported the menstrual cycle phase while others were even more strict and performed the evaluations when participants were in a specific phase. For example, Buck et al. [26] standardized the assessments with the protocol of starting in the first three days after the last menstruation (follicular phase), Chen et al. [34] instructed athletes to participate during their early follicular phase and Puente et al. [14], Stojanovic et al. [38] and Karayigit et al. [46] completed their assessments during the luteal phase. Specific phases and the use of oral contraceptives are specified in Table 1.

\subsection{Rested, Match and Fatigued Conditions}

For the rested and match conditions, enough studies were included to perform a metaanalysis, as presented below. For the fatigued condition, meta-analyses of RPE, agility, RSA and maximal voluntary isometric contraction (MVIC) were performed. We could not perform a meta-analysis for the other fitness tests in a fatigued condition due to the 
heterogeneity of the performed tests. Nonetheless, the last column of Table 1 presents three different symbols reflecting the effectiveness of caffeine supplementation in each individual study, with an up-arrow representing a positive effect. Of the 18 included studies, 10 performed tests including a fatigued state. Of these 10 studies, a total of 46 variables were analyzed after participants were already fatigued, finding a positive effect of caffeine for only 8 variables, as specified in the last column of Table 1.

\subsection{Meta-Analysis Results}

\subsubsection{Simulated Match Body Impacts}

Four studies evaluated body impacts during a match, including studies of volleyball [30], basketball [14], handball [32] and rugby players [31]. The meta-analysis including total body impacts is presented in Figure 2A and shows that caffeine did improve intensity during a match (standardized mean difference (SMD): 0.488; 95\% CI: $0.050,0.927$ ). Heterogeneity among the studies was low $\left(\mathrm{I}^{2} 49 \%, p=0.117\right)$.

\subsubsection{Specific Sport Drills}

Four studies analyzed a specific sport movement (Figure 2B). On the one hand, PerezLopez et al. [30] analyzed the speed of a volleyball ball in a jumping spike, while Muñoz et al. analyzed the speed of a $9 \mathrm{~m}$ handball throw against a goalkeeper. On the other hand, Puente et al. [14] and Tan et al. [35] measured basketball throw performance. Caffeine improved performance on overall specific sport drills (SMD: 0.384; 95\% CI: 0.077, 0.691). Heterogeneity among the studies was low $\left(\mathrm{I}^{2} 0, p=0.699\right)$. A subgroup meta-analysis was performed, showing that caffeine improved ball speed (SMD: 0.440; 95\% CI: 0.098; $\mathrm{I}^{2} 0 \%$ $p=0.903$ ) but did not improve effectiveness during basketball free throws (SMD: 0.150, $95 \%$ CI: $\left.-0.549,0.849 ; \mathrm{I}^{2} 0 \% p=0.347\right)$.

\subsubsection{Jump Performance}

Seven studies evaluated jump performance using either countermovement jumps (CMJ), Abalakov jumps (ABA) or squat jumps (SJ). One study did not describe the jump performed, calling it a vertical jump [33]. As shown in Figure 2C, caffeine showed a positive effect on CMJ performance (SMD: $0.208,95 \%$ CI: $0.079,0.338 ; I^{2} 0 \% p=0.989$ ). The sensitivity analysis excluding the Fernandez-Campos study due to the supplied dosage of caffeine $(<2 \mathrm{mg} / \mathrm{kg}$ ) revealed similar results (SMD: $0.217,95 \%$ CI: $0.085,0.348)$ with a low heterogeneity $\left(\mathrm{I}^{2} 0 \% p=0.994\right)$.

Another meta-analysis was performed including three studies that measured SJ with the intake of caffeine showing no improvement in SJ performance (Figure 2D: SMD: 0.241, $95 \%$ CI: $\left.-0.189,0.671 ; I^{2} 0 \% p=0.870\right)$. The sensitivity analysis excluding the FernandezCampos study revealed similar results (SMD: $0.345,95 \%$ CI: $-0.237,0.928 ; \mathrm{I}^{2} 0 \% p=0.926$ ).

\subsubsection{Agility}

From the six studies that evaluated agility in a rested state, three used the $t$-test $[27,30,40]$, while one study used a modified version of the t-test [32], one study used the change-ofdirection and acceleration test (CODAT) [14] and one study used the lane agility drill [38]. Figure $3 \mathrm{~A}$ presents the performed meta-analysis including all the agility tests, showing that caffeine did not improve agility (SMD: $0.144,95 \%$ CI: $-0.127,0.416 ; \mathrm{I}^{2} 0 \% p=0.939$ ). 


\section{Studies}

Perez-Lopez 2015

Puente 2017

Munoz 2020

Portillo 2017

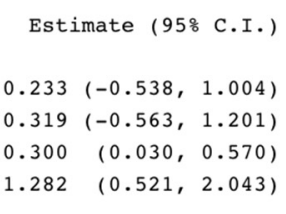

Overall $\left(I^{\wedge} 2=4910 \%, P=0.117\right) \quad 0.488 \quad(0.050,0.927)$

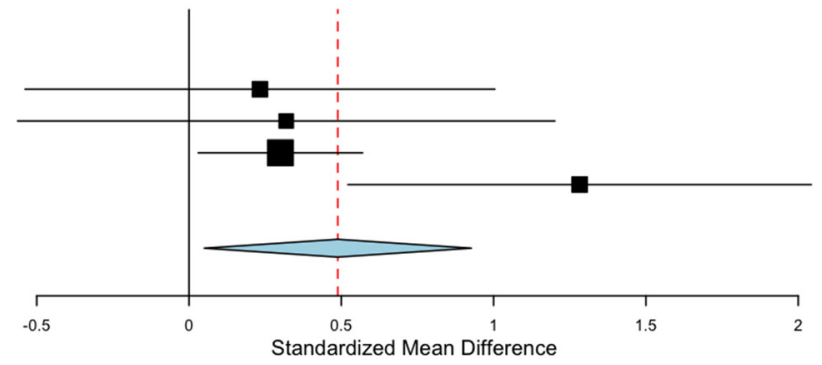

(A)

\begin{tabular}{|c|c|}
\hline Studies & Estimate (958 C.I.) \\
\hline Perez-Lopez 2015 & $0.396(-0.380,1.172)$ \\
\hline Munoz 2020 & $0.450 \quad(0.070,0.830)$ \\
\hline Subgroup ball speed $\left(I^{\wedge} 2=0 \%, P=0.903\right)$ & $0.440 \quad(0.098,0.781)$ \\
\hline Puente 2017 & $0.411(-0.475,1.297)$ \\
\hline Tan 2020 & $-0.280(-1.417,0.857)$ \\
\hline Subgroup free throw $\left(1^{\wedge} 2=0 \%, P=0.347\right)$ & $0.150(-0.549,0.849)$ \\
\hline Derall $\left(I^{\wedge} 2=0 \%, P=0.699\right)$ & $0.384 \quad(0.077,0.691)$ \\
\hline
\end{tabular}

\begin{tabular}{lrr} 
Studies & \multicolumn{2}{c}{ Estimate $(95$ C.I. $)$} \\
Perez-Lopez 2015 & 0.234 & $(-0.537,1.005)$ \\
Stojanovic 2019 & 0.284 & $(-0.597,1.165)$ \\
Munoz 2020 & 0.220 & $(0.080,0.360)$ \\
Fernandez-Campos 2015 & $0.020(0.616,0.656)$ \\
Ali, a 2016 & 0.019 & $(-0.857,0.896)$ \\
Lara 2014 & $0.200(-0.454,0.855)$ \\
Overall (I^2=0\%, P=0.989) & $\mathbf{0 . 2 0 8}$ & $(\mathbf{0 . 0 7 9}, \mathbf{0 . 3 3 7})$
\end{tabular}

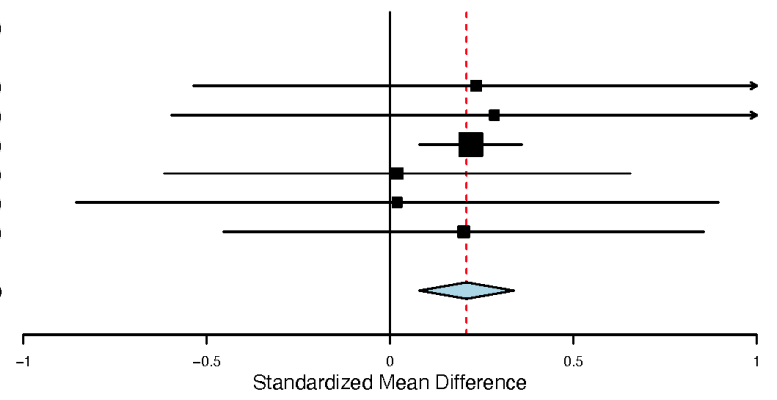

(C)

\section{Studies}

Perez-Lopez 2015

Stojanovic 2019

Fernandez-Campos 2015

Overall $\left(\left.\right|^{\wedge} 2=0 \%, P=0.870\right) \quad 0.241(-0.189,0.671)$
(B)

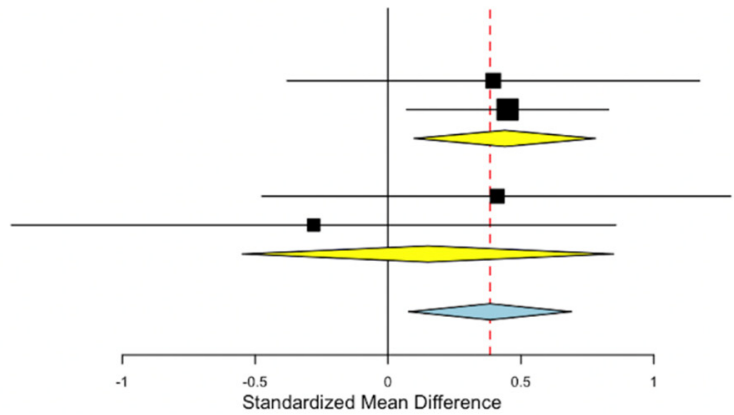

(D)

Figure 2. (A): Effects of caffeine on body impacts during a simulated match. (B): Effects of caffeine on specific skills. (C): Effects of caffeine on countermovement jump. (D): Effects of caffeine on squat jump. 

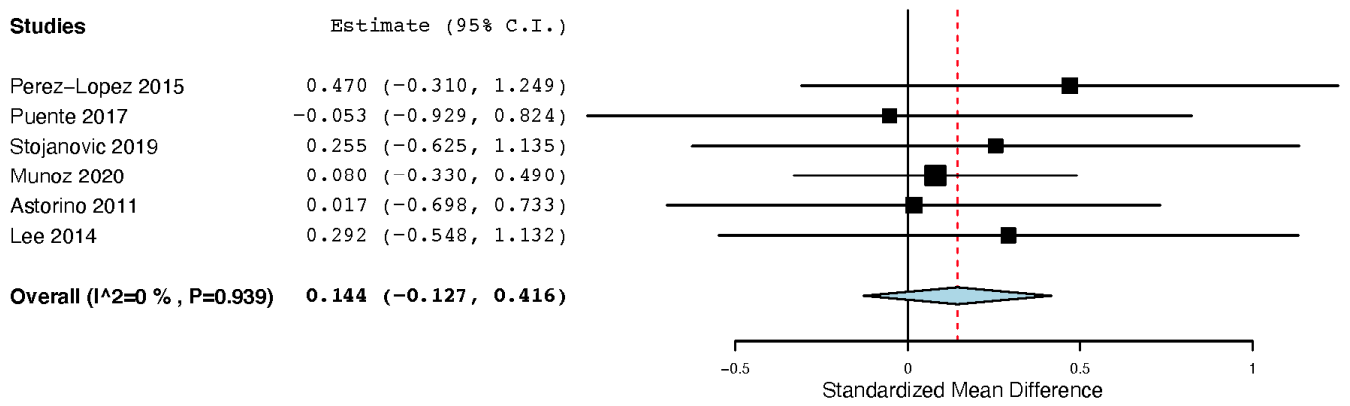

(A)
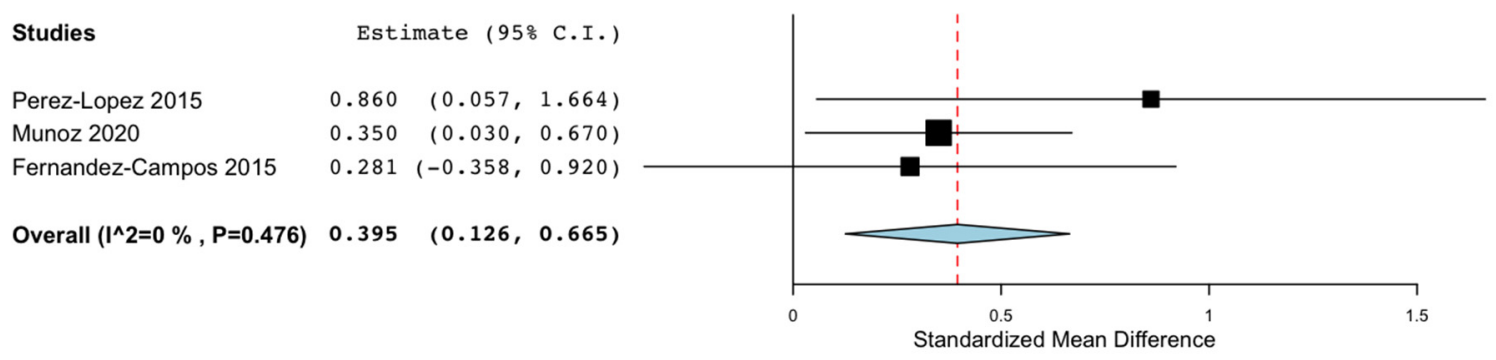

(B)

Figure 3. (A): Effects of caffeine on agility. (B): Effects of caffeine on handgrip strength.

The sensitivity analysis excluding the Astorino et al. study showed similar results (SMD: $0.166,95 \%$ CI: $-0.128,0.459 ; \mathrm{I}^{2} 0 \% p=0.892$ ).

\subsubsection{Handgrip}

Figure 3B shows the effects of caffeine on handgrip strength, which was measured in three studies, with Muñoz [32] reporting the mean strength of both hands in handball players, while Perez-Lopez [30] and Fernandez-Campos [41] reported separated values for the left and right hands of volleyball players (the right hand was selected for the present meta-analysis as it is usually the dominant hand). The meta-analysis showed that caffeine had a positive effect on handgrip performance (SMD: 0.395, 95\% CI: 0.126, 0.665). A low heterogeneity was found $\left(\mathrm{I}^{2} 0 \% p=0.476\right)$. A sensitivity analysis excluding the FernandezCampos study did not change the main effect of caffeine (SMD: 0.467, 95\% CI: 0.047, 0.887) or the low heterogeneity $\left(\mathrm{I}^{2} 25 \% p=0.238\right)$.

\subsubsection{Single Sprint Performance}

Single sprint performance was evaluated in five studies, from which two performed a single sprint $[32,38]$ and three performed an RSA test, with the first sprint selected for the present meta-analysis $[26,28,29]$. A subgroup meta-analysis was performed for studies that used a single sprint on the one hand and for studies that used the first sprint of an RSA test on the other hand. All the studies performed a $30 \mathrm{~m}$ sprint except for that of Stojanović and colleagues [38] who used a $20 \mathrm{~m}$ sprint. As presented in Figure 4A, caffeine showed no effect on single sprint performance (SMD: 0.225, 95\% CI: $-0.022,0.472 ; \mathrm{I}^{2} 0 \%$ $p=0.685$ ). Nonetheless, when dividing studies into two groups according to the type of measurement performed (single sprint or first sprint of an RSA test), we found that those studies that performed a single sprint reported a performance improvement (SMD: 0.347, 95\% CI: $0.038,0.656 ; \mathrm{I}^{2} 0 \% p=0.903$ ), while in those studies that performed a RSA test, caffeine did not improve the performance of the first sprint (SMD: 0.011, 95\% CI: -0.399 , $\left.0.420 ; I^{2} 0 \% p=0.737\right)$. 


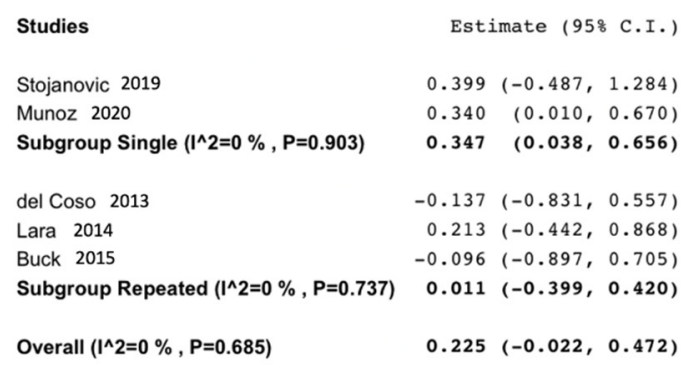

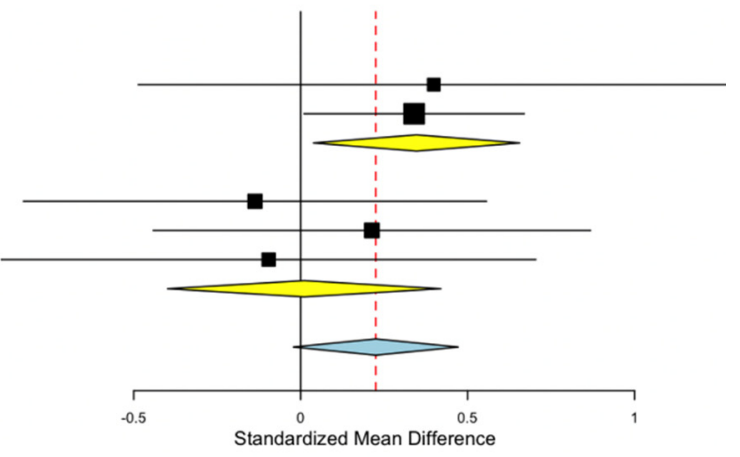

(A)

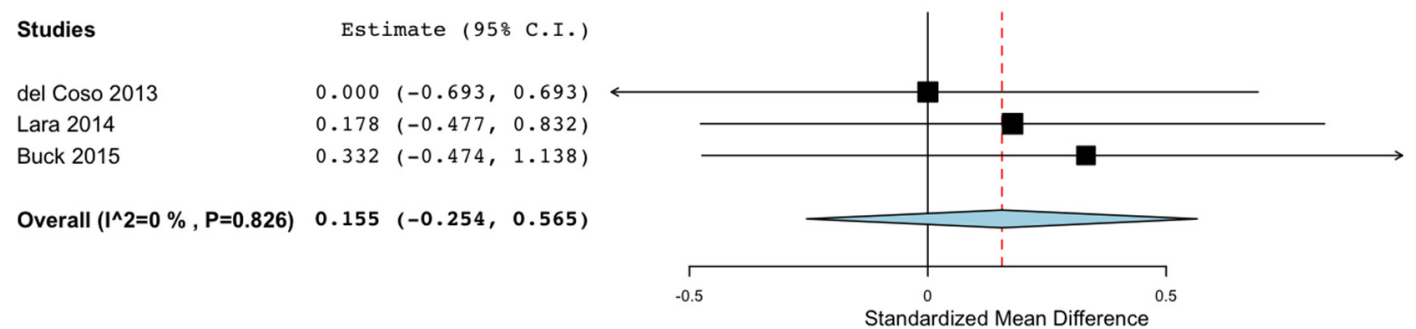

(B)

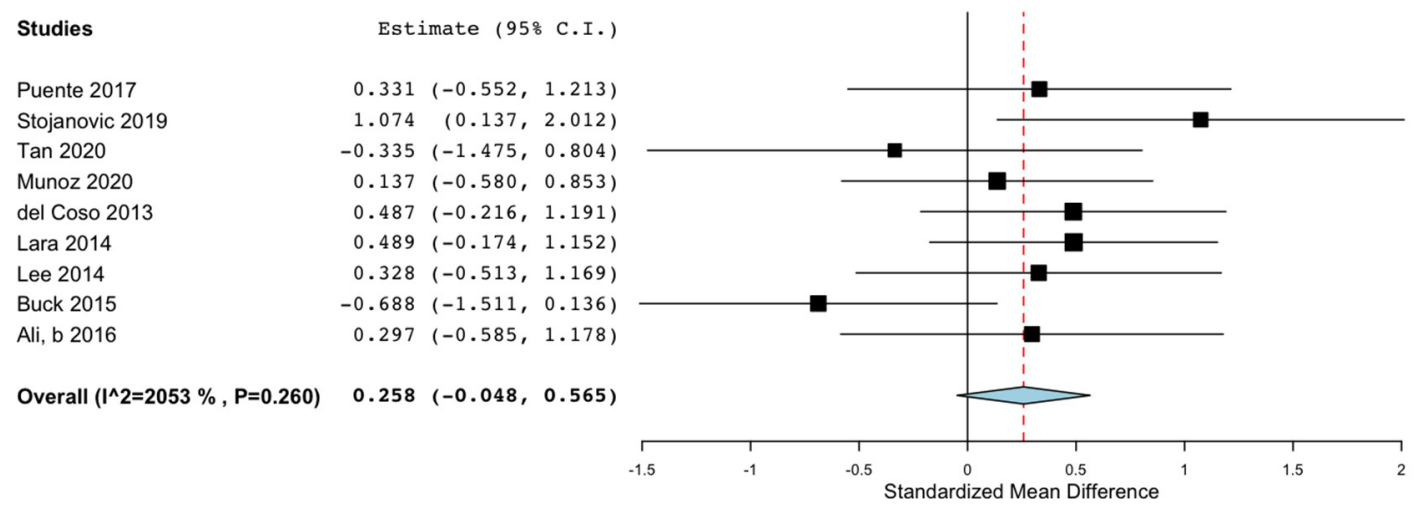

(C)

Figure 4. (A): Effects of caffeine on single sprint performance. (B): Effects of caffeine on repeated sprint ability performance. (C): Effects of caffeine on rate of perceived exertion.

\subsubsection{RSA}

Three studies included an RSA test, with Del Coso et al. [28] using a $6 \times 30 \mathrm{~m}$ RSA test, Lara et al. [29] using a $7 \times 30 \mathrm{~m}$ RSA test and Buck et al. [26] using a $6 \times 20 \mathrm{~m}$ RSA test. As shown in Figure 4B, caffeine showed no effect on RSA (SMD: 0.155, 95\% CI: -0.254, $0.565 ; \mathrm{I}^{2} 0 \% p=0.826$ ). 


\subsubsection{RPE}

As shown in Figure 4C, nine studies evaluated RPE after performing an exercise protocol after caffeine supplementation, finding no effect of this supplement on RPE (SMD: $0.258,95 \%$ CI: $\left.-0.048,0.565 ; \mathrm{I}^{2} 0.26 \% p=0.260\right)$.

\subsubsection{Fatigued State}

Six studies performed the assessments after applying a specific fatigue protocol or after a match. A meta-analysis could only be performed for the agility tests, as three studies evaluated agility after performing several all-out sprint tests $[27,40]$ or after a match [33], with the pooled results showing no positive effects on agility after fatiguing the participants, as shown in Figure 5 (SMD: 0.069, 95\% CI: $-0.400,0.538 ; \mathrm{I}^{2} 0 \% p=0.858$ ). It should be highlighted that the caffeine doses for two of the aforementioned studies were below $2 \mathrm{mg} / \mathrm{kg}[33,40]$.
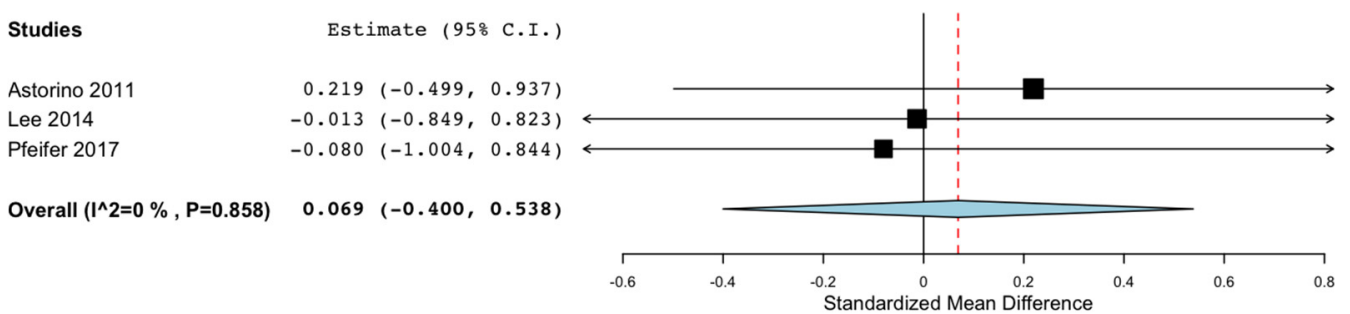

Figure 5. Effects of caffeine on agility after a fatigue protocol.

\section{Discussion}

The main findings of the present systematic review and meta-analysis suggest that oral caffeine administration before exercise has an ergogenic effect on specific team-sport skills, CMJ height, handgrip strength and total body impacts in female TSA. Nonetheless, caffeine did not show an ergogenic effect on RPE, SJ, agility, RSA or tests performed in a fatigued state.

The positive effects of caffeine supplementation on CMJ are in accordance with most of the previous systematic reviews and meta-analyses developed for team sports [8-10], although Ferreira and colleagues [11] did not find a positive effect of caffeine in their meta-analysis including male soccer players. Nonetheless, we also found that caffeine had no effect on SJ performance. This could partially be explained by the low number of studies $(n=3)$ evaluating the effect of caffeine on SJ performance. When considering Figure 2D, it appears that caffeine had a positive effect in the three studies, although the overall effect was not significant probably due to the low number of studies.

Muscle force and consequently CMJ could both determine specific athletic skills performance, which is of critical importance for TSA and was found to be improved by caffeine supplementation. Nonetheless, we only found a positive effect for the subgroup meta-analysis that included ball speed, which might be influenced by technique and upperbody strength/power. This would suggest that caffeine could be useful for those team sports in which upper-body strength/power is a determinant (e.g., volleyball, basketball or handball). Nevertheless, these results should be interpreted with caution, as only two studies measured ball speed, and the two studies that evaluated accuracy (through basketball free throws) found no ergogenic effects [14,35].

The improvement in CMJ performance was accompanied by an improvement in single sprint performance (when studies that only performed one sprint were included). This is in line with previous systematic reviews and meta-analyses, which found positive results in single sprints developed with TSA [8,9]. When we included the first sprint of studies that performed an RSA test the ergogenic effect of caffeine disappeared. We could therefore hypothesize that those participants who were going to complete an RSA test might not have performed the first sprint at their maximal capacity, and that caffeine supplementation 
does indeed have a positive effect on single sprint performance when participants are performing a single maximal-effort sprint.

Although single sprint performance is important, most team-sport athletes will need to perform several sprints during a match with short low-intensity periods between them. Consequently, several studies performed RSA tests in order to evaluate the ability of athletes to maintain sprint intensity. We found that caffeine supplementation had no effect on RSA in our meta-analysis, which disagrees with some previous systematic reviews and meta-analyses carried out with samples of men and women involved in team sports $[9,10]$ but is in line with others $[7,11]$. Again, a small number of studies were included in our meta-analysis $(n=3)$, and therefore the results should be interpreted with caution, as more studies including female TSA are necessary.

The positive findings in upper-limbs isometric muscle force are in line with a recent meta-analysis developed by Grgic and Del Coso [47] focusing on the effects of caffeine on strength and power, finding that caffeine improved upper-body performance in women. This could be critical for female TSA, as an improvement in muscular endurance and strength could enable TSA to develop improved performance during a match. Along these lines, we did find improvements in total body impacts (a proxy for the players' match intensity), which would imply higher intensities during competition. Consequently, although athletes might not be able to improve RSA under laboratory conditions, they might be more motivated during a match and be capable of improving intensity due to the ergogenic effect of caffeine. These positive findings are in line with previous meta-analyses that found improvements in the performed number of sprints during a real or simulated match after acute caffeine ingestion [9].

The lack of effect of caffeine ingestion on agility tests was surprising and contradicted results from a previous meta-analysis which included mainly male participants (two studies evaluated females out of eight studies included) [9]. These contrasting results highlight the importance of performing more research with female athletes, as the scientific community may be assuming that what works with males will work in exactly the same way with females, while we have found some differences in the current meta-analysis.

Regarding RPE, our findings are similar to those of previous meta-analyses developed for team sports $[9,11]$ which found no effects of caffeine on RPE. These studies and ours, which are all focused on team sports, show opposite results to those found in a larger meta-analysis developed in 2005 [48] which found a $6 \%$ reduction in RPE after the ingestion of caffeine in endurance tests. In the 21 included studies (where 7 measured females), there were 13 cycling tests, 5 running tests, 2 rowing tests and 1 swimming test. It is important to notice that the aforementioned meta-analysis showed a reduction in RPE only when constant loads were applied. Team sports are characterized by numerous high-intensity efforts followed by rest periods and do not follow a constant load pattern, which could explain the lack of effect of caffeine on RPE found in the present meta-analysis. Nonetheless, this may only be partially true, as a previous study [48] also found that, although no differences were found at the end of a test to exhaustion, caffeine attenuated RPE during exercise, which could partially explain the performance improvements found in some athletes. Most of the studies included in our meta-analysis only included an RPE assessment at the end of the tests or matches, but it would be interesting for future studies to consider RPE throughout exercise. This would allow researchers to test if a reduced RPE, and therefore an increased physical performance for the same intensity, is found during a match or a laboratory test.

Along the same lines, it would be interesting to evaluate the effects of caffeine in fatigued conditions, as many of the presented studies in the current meta-analysis were developed in laboratory settings and included participants in a rested state who performed the test (agility, jumps, etc.) $60 \mathrm{~min}$ after the ingestion of caffeine. Nonetheless, given that TSA are usually exposed to fatiguing efforts, it would be interesting to develop more studies in fatigued conditions, as caffeine presents the ability to cross the blood-brain barrier and block the adenosine receptors in the brain, mitigating the negative effects of 
fatigue. Very few studies have evaluated the effects of caffeine after applying a fatigue protocol ( $n=3,2$ and 2 for agility, RSA and MVIC, respectively). The only meta-analysis performed showed a lack of effect of caffeine on agility performance when participants were fatigued. Nonetheless, caffeine doses for two $[33,40]$ of the three studies included were below $2 \mathrm{mg} / \mathrm{kg}$, and therefore further studies are required to evaluate the effects of higher doses in fatigued female TSA. Although we could not perform a further metaanalysis including the fatigued state, the results from Table 1 suggest a lack of effect as the last column shows that out of the 46 variables that were registered in fatigued conditions, only 8 improved after caffeine ingestion.

In order to improve research in this topic, we would encourage future studies to report the menstrual cycle phase of participants when performing the experiments and to perform both placebo and supplementation trials during the same menstrual cycle phase, in order to reduce the possible effect of the menstrual cycle phase. The use of oral contraceptives should also be registered. Experiments should test both rested and fatigued conditions, register individual responses to caffeine which were not reported in most of the studies included in the present meta-analysis and evaluate if in "responders" lower dosages have the same effect or if increasing the dose in "non-responders" has a positive effect. This is because previous articles have identified substantial inter-individual variations following caffeine ingestion in sport [49]. These differences seem to be mediated by genetic variations, and the characterization of the athlete's genetic profile could potentially help in individualizing the caffeine dose accurately to optimize its effect on physical performance [49].

Finally, it is worth mentioning that five of the included studies used energy drinks that, in addition to caffeine, contained other substances that could also have an ergogenic effect, such as sugar, glucuronolactone and taurine. Nonetheless, three [28-30] of these studies specified that the placebo drink and the energy drink were exactly the same drinks with the only difference being the caffeine content (the placebo had $0 \mathrm{mg} / \mathrm{kg}$ ). The two remaining studies were those developed by Astorino et al. [40] and by Fernandez-Campos et al. [41]. Astorino et al. [40] used an energy drink containing both taurine and glucuronolactone. The taurine content was $1 \mathrm{~g}$, which is far from the $6 \mathrm{~g}$ suggested to have an ergogenic effect [50,51]. In the case of glucuronolactone, as stated by Campos-Perez in the book Sports and Energy drinks: "because of the few investigations on the isolate glucuronolactone in humans, there is no evidence to support the idea of adding this compound to energy drinks to improve physical and sport performance, not even as a complement to the action of taurine and/or caffeine" [52]. Therefore, the only added ingredient that could make a difference in the included studies and influence performance was sugar, with the Astorino et al. [40] study showing a difference of $7 \mathrm{~g}$ (energy drink $27 \mathrm{~g}$ vs. placebo $20 \mathrm{~g}$ of sugar) and the Fernandez-Campos et al. [41] study showing a difference of $31 \mathrm{~g}$ (energy drink $31 \mathrm{~g}$ vs. placebo $0 \mathrm{~g}$ of sugar). Nonetheless, both studies were included in the sensitivity analyses, and we consequently repeated the meta-analyses without including them, finding similar results. Therefore, the possible effect that other ergogenic substances might have that could enhance the findings attributed to caffeine were controlled for in the present meta-analysis.

Although the present meta-analysis presents several strengths, such as the focus on an athlete population with limited previous scientific evidence (female TSA), the effort to contact the corresponding authors to obtain specific data for this group and the inclusion of the updated 2021 PRISMA guidelines, it is not without limitations, the main one being the low number of studies included in some of the meta-analyses $(n=3)$.

\section{Conclusions}

Although caffeine is generally considered as one of the most useful supplements used to increase athletic performance, the results of the present meta-analysis suggest that more research is needed in female TSA. Female TSA obtained benefits from caffein supplementation as it was shown to improve upper-body strength and sport-specific tasks 
related to upper-body strength (ball speed), in addition to CMJ, single sprint performance and body impacts during a match (match intensity).

Supplementary Materials: The following are available online at https://www.mdpi.com/article/ 10.3390/nu13103663/s1, Table S1: Search strategy; Table S2: Quality Assessment of included randomized controlled trials (PEDro scores); Table S3: Quality Assessment of included randomized controlled trials (RoB 2 tool).

Author Contributions: Conceptualization: A.G.-B. and G.L.-B.; methodology, A.G.-B., G.L.-B., J.M.-P., B.M.-P.; formal analysis, A.G.-B. and J.M.-P.; resources, A.G.-C.; writing—original draft preparation, A.G.-B. and G.L.-B.; writing-review and editing, J.D.C., J.A.C., A.M.-L., G.V.-R., A.G.-C., J.M.-P. and B.M.-P.; funding acquisition, A.G.-B. and A.G.-C. All authors have read and agreed to the published version of the manuscript.

Funding: The APC was funded by “Proyectos Unizar CUD, 2020”: UZCUD2020-BIO-02.

Institutional Review Board Statement: Not applicable.

Informed Consent Statement: Not applicable.

Data Availability Statement: Not applicable.

Acknowledgments: The present research has been possible thanks to the funding obtained from "Proyectos Unizar CUD, 2020": UZCUD2020-BIO-02.

Conflicts of Interest: The authors declare no conflict of interest.

\section{References}

1. Del Coso, J.; Muñoz, G.; Muñoz-Guerra, J. Prevalence of caffeine use in elite athletes following its removal from the World Anti-Doping Agency list of banned substances. Appl. Physiol. Nutr. Metab. 2011, 36, 555-561. [CrossRef] [PubMed]

2. WADA. Monitoring Program; World Anti-Doping Agency: Montreal, QC, Canada, 2020.

3. Aguilar-Navarro, M.; Muñoz, G.; Salinero, J.J.; Muñoz-Guerra, J.; Fernández-Álvarez, M.; Plata, M.D.M.; Del Coso, J. Urine Caffeine Concentration in Doping Control Samples from 2004 to 2015. Nutrients 2019, 11, 286. [CrossRef] [PubMed]

4. Kerksick, C.M.; Wilborn, C.D.; Roberts, M.D.; Smith-Ryan, A.; Kleiner, S.M.; Jäger, R.; Collins, R.; Cooke, M.; Davis, J.N.; Galvan, E.; et al. ISSN exercise \& sports nutrition review update: Research \& recommendations. J. Int. Soc. Sports Nutr. 2018, 15, 38. [CrossRef] [PubMed]

5. Grgic, J.; Grgic, I.; Pickering, C.; Schoenfeld, B.J.; Bishop, D.J.; Pedisic, Z. Wake up and smell the coffee: Caffeine supplementation and exercise performance-An umbrella review of 21 published meta-analyses. Br. J. Sports Med. 2019, 54, 681-688. [CrossRef]

6. Southward, K.; Rutherfurd-Markwick, K.J.; Ali, A. The Effect of Acute Caffeine Ingestion on Endurance Performance: A Systematic Review and Meta-Analysis. Sports Med. 2018, 48, 1913-1928. [CrossRef]

7. Brown, S.J.; Brown, J.; Foskett, A. The Effects of Caffeine on Repeated Sprint Performance in Team Sport Athletes—A MetaAnalysis. Sport Sci. Rev. 2013, 22, 25-32. [CrossRef]

8. Chia, J.S.; Barrett, L.A.; Chow, J.Y.; Burns, S.F. Effects of Caffeine Supplementation on Performance in Ball Games. Sports Med. 2017, 47, 2453-2471. [CrossRef]

9. Salinero, J.J.; Lara, B.; Del Coso, J. Effects of acute ingestion of caffeine on team sports performance: A systematic review and meta-analysis. Res. Sports Med. 2019, 27, 238-256. [CrossRef]

10. Mielgo-Ayuso, J.; Calleja-Gonzalez, J.; Del Coso, J.; Urdampilleta, A.; León-Guereño, P.; Fernández-Lázaro, D. Caffeine Supplementation and Physical Performance, Muscle Damage and Perception of Fatigue in Soccer Players: A Systematic Review. Nutrients 2019, 11, 440. [CrossRef]

11. Ferreira, R.E.S.; Pacheco, R.L.; de Oliveira Cruz Latorraca, C.; Riera, R.; Eid, R.G.; Martimbianco, A.L.C. Effects of Caffeine Supplementation on Physical Performance of Soccer Players: Systematic Review and Meta-Analysis. Sports Health 2021, 13, 347-358. [CrossRef]

12. Salinero, J.J.; Lara, B.; Jim 'énez-Ormeño, E.; Romero-Moraleda, B.; Giráldez-Costas, V.; Baltazar-Martins, G.; Del Coso, J. More Research Is Necessary to Establish the Ergogenic Effect of Caffeine in Female Athletes. Nutrients 2019, 11, 1600. [CrossRef]

13. Bello, M.L.; Walker, A.J.; McFadden, B.A.; Sanders, D.J.; Arent, S.M. The effects of TeaCrine ${ }^{\circledR}$ and caffeine on endurance and cognitive performance during a simulated match in high-level soccer players. J. Int. Soc. Sports Nutr. 2019, 16, 20. [CrossRef]

14. Puente, C.; Abián-Vicén, J.; Salinero, J.J.; Lara, B.; Areces, F.; Del Coso, J. Caffeine Improves Basketball Performance in Experienced Basketball Players. Nutrients 2017, 9, 1033. [CrossRef]

15. Maughan, R.J.; Burke, L.M.; Dvorak, J.; Larson-Meyer, D.E.; Peeling, P.; Phillips, S.M.; Rawson, E.S.; Walsh, N.P.; Garthe, I.; Geyer, H.; et al. IOC consensus statement: Dietary supplements and the high-performance athlete. Br. J. Sports Med. 2018, 52, 439-455. [CrossRef] 
16. Lara, B.; Hellín, J.G.; Ruiz-Mereno, C.; Romero-Moraleda, B.; Del Coso, J. Acute caffeine intake increases performance in the 15-s Wingate test during the menstrual cycle. Br. J. Clin. Pharmacol. 2020, 86, 745-752. [CrossRef]

17. Guest, N.S.; VanDusseldorp, T.A.; Nelson, M.T.; Grgic, J.; Schoenfeld, B.J.; Jenkins, N.D.M.; Arent, S.M.; Antonio, J.; Stout, J.R.; Trexler, E.T.; et al. International society of sports nutrition position stand: Caffeine and exercise performance. J. Int. Soc. Sports Nutr. 2021, 18, 1. [CrossRef]

18. Temple, J.L.; Ziegler, A.M. Gender Differences in Subjective and Physiological Responses to Caffeine and the Role of Steroid Hormones. J. Caffeine Res. 2011, 1, 41-48. [CrossRef]

19. Sabblah, S.; Dixon, D.; Bottoms, L. Sex differences on the acute effects of caffeine on maximal strength and muscular endurance. Comp. Exerc. Physiol. 2015, 11, 89-94. [CrossRef]

20. Skinner, T.; Desbrow, B.; Arapova, J.; Schaumberg, M.A.; Osborne, J.; Grant, G.D.; Anoopkumar-Dukie, S.; Leveritt, M.D. Women Experience the Same Ergogenic Response to Caffeine as Men. Med. Sci. Sports Exerc. 2019, 51, 1195-1202. [CrossRef]

21. Lara, B.; Salinero, J.J.; Giráldez-Costas, V.; Del Coso, J. Similar ergogenic effect of caffeine on anaerobic performance in men and women athletes. Eur. J. Nutr. 2021, 60, 4107-4114. [CrossRef]

22. Chen, H.-Y.; Chen, Y.-C.; Tung, K.; Chao, H.-H.; Wang, H.-S. Effects of caffeine and sex on muscle performance and delayed-onset muscle soreness after exercise-induced muscle damage: A double-blind randomized trial. J. Appl. Physiol. 2019, 127, 798-805. [CrossRef]

23. Page, M.J.; McKenzie, J.E.; Bossuyt, P.M.; Boutron, I.; Hoffmann, T.C.; Mulrow, C.D.; Shamseer, L.; Tetzlaff, J.M.; Akl, E.A.; Brennan, S.E.; et al. The PRISMA 2020 statement: An updated guideline for reporting systematic reviews. BMJ 2021, 372, n71. [CrossRef]

24. Maher, C.G.; Sherrington, C.; Herbert, R.D.; Moseley, A.M.; Elkins, M. Reliability of the PEDro Scale for Rating Quality of Randomized Controlled Trials. Phys. Ther. 2003, 83, 713-721. [CrossRef]

25. Sterne, J.A.C.; Savović, J.; Page, M.; Elbers, R.G.; Blencowe, N.; Boutron, I.; Cates, C.; Cheng, H.-Y.; Corbett, M.S.; Eldridge, S.M.; et al. RoB 2: A revised tool for assessing risk of bias in randomised trials. BMJ 2019, 366, 14898. [CrossRef]

26. Kopec, B.J.; Dawson, B.T.; Buck, C.; Wallman, K.E. Effects of sodium phosphate and caffeine ingestion on repeated-sprint ability in male athletes. J. Sci. Med. Sport 2016, 19, 272-276. [CrossRef]

27. Lee, C.-L.; Cheng, C.-F.; Astorino, T.A.; Lee, C.J.; Huang, H.-W.; Chang, W.-D. Effects of carbohydrate combined with caffeine on repeated sprint cycling and agility performance in female athletes. J. Int. Soc. Sports Nutr. 2014, 11, 17. [CrossRef]

28. Del Coso, J.; Portillo, J.; Muñoz, G.; Abián-Vicén, J.; Gonzalez-Millán, C.; Muñoz-Guerra, J. Caffeine-containing energy drink improves sprint performance during an international rugby sevens competition. Amino Acids 2013, 44, 1511-1519. [CrossRef]

29. Lara, B.; González-Millán, C.; Salinero, J.J.; Abián-Vicén, J.; Areces, F.; Barbero-Alvarez, J.C.; Muñoz, V.; Portillo, L.J.; GonzalezRave, J.M.; Del Coso, J. Caffeine-containing energy drink improves physical performance in female soccer players. Amino Acids 2014, 46, 1385-1392. [CrossRef]

30. Pérez-López, A.; Salinero, J.J.; Abián-Vicén, J.; Valadés, D.; Lara, B.; Hernandez, C.; Areces, F.; González, C.; Del Coso, J. Caffeinated Energy Drinks Improve Volleyball Performance in Elite Female Players. Med. Sci. Sports Exerc. 2015, 47, 850-856. [CrossRef]

31. Portillo, J.; Del Coso, J.; Abián-Vicén, J. Effects of Caffeine Ingestion on Skill Performance During an International Female Rugby Sevens Competition. J. Strength Cond. Res. 2017, 31, 3351-3357. [CrossRef]

32. Muñoz, A.; López-Samanes, A.; Pérez-López, A.; Aguilar-Navarro, M.; Moreno-Heredero, B.; Rivilla-García, J.; González-Frutos, P.; Pino-Ortega, J.; Morencos, E.; Del Coso, J. Effects of Caffeine Ingestion on Physical Performance in Elite Women Handball Players: A Randomized, Controlled Study. Int. J. Sports Physiol. Perform. 2020, 15, 1406-1413. [CrossRef] [PubMed]

33. Pfeifer, D.R.; Arvin, K.M.; Herschberger, C.N.; Haynes, N.J.; Renfrow, M.S. A Low Dose Caffeine and Carbohydrate Supplement does not Improve Athletic Performance during Volleyball Competition. Int. J. Exerc. Sci. 2017, 10, 340-353. [PubMed]

34. Chen, H.-Y.; Wang, H.-S.; Tung, K.; Chao, H.-H. Effects of Gender Difference and Caffeine Supplementation on Anaerobic Muscle Performance. Int. J. Sports Med. 2015, 36, 974-978. [CrossRef] [PubMed]

35. Tan, Z.S.; Burns, S.F.; Pan, J.W.; Kong, P.W. Effect of caffeine ingestion on free-throw performance in college basketball players. J. Exerc. Sci. Fit. 2020, 18, 62-67. [CrossRef]

36. Scanlan, A.T.; Dalbo, V.J.; Conte, D.; Stojanović, E.; Stojiljković, N.; Stanković, R.; Antić, V.; Milanović, Z. No Effect of Caffeine Supplementation on Dribbling Speed in Elite Basketball Players. Int. J. Sports Physiol. Perform. 2019, 14, 997-1000. [CrossRef]

37. Muñoz, A.; López-Samanes, A.; Aguilar-Navarro, M.; Varillas-Delgado, D.; Rivilla-García, J.; Moreno-Pérez, V.; Del Coso, J. Effects of CYP1A2 and ADORA2A Genotypes on the Ergogenic Response to Caffeine in Professional Handball Players. Genes 2020, 11, 933. [CrossRef]

38. Stojanovic, E.; Stojiljkovic, N.; Scanlan, A.T.; Dalbo, V.J.; Stankovic, R.; Antic, V.; Milanovic, Z. Acute caffeine supplementation promotes small to moderate improvements in performance tests indicative of in-game success in professional female basketball players. Appl. Physiol. Nutr. Metab. 2019, 44, 849-856. [CrossRef]

39. Higgins, J.P.T.; Thomas, J.; Chandler, J.; Cumpston, M.; Li, T.; Page, M.J.; Welch, V.A. Cochrane Handbook for Systematic Reviews of Interventions; Higgins, J.P.T., Thomas, J., Chandler, J., Cumpston, M., Li, T., Page, M.J., Welch, V.A., Eds.; Wiley: Hoboken, NJ, USA, 2019; ISBN 9781119536628.

40. Astorino, T.A.; Matera, A.J.; Basinger, J.; Evans, M.; Schurman, T.; Marquez, R. Effects of red bull energy drink on repeated sprint performance in women athletes. Amino Acids 2012, 42, 1803-1808. [CrossRef] 
41. Fernández-Campos, C.; Dengo, A.L.; Moncada-Jiménez, J. Acute Consumption of an Energy Drink Does Not Improve Physical Performance of Female Volleyball Players. Int. J. Sport Nutr. Exerc. Metab. 2015, 25, 271-277. [CrossRef]

42. Higgins, J.P.T.; Thompson, S.G.; Deeks, J.; Altman, D.G. Measuring inconsistency in meta-analyses. BMJ 2003, 327, 557-560. [CrossRef]

43. Mahdavi, R.; Daneghian, S.; Jafari, A.; Homayouni, A. Effect of Acute Caffeine Supplementation on Anaerobic Power and Blood Lactate Levels in Female Athletes. J. Caffeine Res. 2015, 5, 83-87. [CrossRef]

44. Ali, A.; O'Donnell, J.; Foskett, A.; Rutherfurd-Markwick, K. The influence of caffeine ingestion on strength and power performance in female team-sport players. J. Int. Soc. Sports Nutr. 2016, 13, 46. [CrossRef]

45. Ali, A.; O’Donnell, J.; Von Hurst, P.; Foskett, A.; Holland, S.; Starck, C.; Rutherfurd-Markwick, K. Caffeine ingestion enhances perceptual responses during intermittent exercise in female team-game players. J. Sports Sci. 2016, 34, 330-341. [CrossRef]

46. Karayigit, R.; Naderi, A.; Akca, F.; Da Cruz, C.J.G.; Sarshin, A.; Yasli, B.C.; Ersoz, G.; Kaviani, M. Effects of Different Doses of Caffeinated Coffee on Muscular Endurance, Cognitive Performance, and Cardiac Autonomic Modulation in Caffeine Naive Female Athletes. Nutrients 2020, 13, 2. [CrossRef]

47. Grgic, J.; Del Coso, J. Ergogenic Effects of Acute Caffeine Intake on Muscular Endurance and Muscular Strength in Women: A Meta-Analysis. Int. J. Environ. Res. Public Health 2021, 18, 5773. [CrossRef]

48. Doherty, M.; Smith, P.M. Effects of caffeine ingestion on rating of perceived exertion during and after exercise: A meta-analysis. Scand. J. Med. Sci. Sports 2005, 15, 69-78. [CrossRef]

49. Pickering, C.; Kiely, J. Are the Current Guidelines on Caffeine Use in Sport Optimal for Everyone? Inter-individual Variation in Caffeine Ergogenicity, and a Move Towards Personalised Sports Nutrition. Sports Med 2018, 48, 7-16. [CrossRef]

50. Zhang, M.; Izumi, I.; Kagamimori, S.; Sokejima, S.; Yamagami, T.; Liu, Z.; Qi, B. Role of taurine supplementation to prevent exercise-induced oxidative stress in healthy young men. Amino Acids 2004, 26, 203-207. [CrossRef]

51. Ward, R.; Bridge, C.A.; McNaughton, L.R.; Sparks, S.A. The effect of acute taurine ingestion on 4-km time trial performance in trained cyclists. Amino Acids 2016, 48, 2581-2587. [CrossRef]

52. Campos-Perez, J.; Camara-Martos, F. Influence of substances present in energy and sports drinks in improving athletic performance. Sports Energy Drinks 2019, 10, 297-337. 